\title{
A search for the cause of cyclical wind variability in $\mathbf{O}$ stars Simultaneous UV and optical observations including magnetic field measurements of the 07.5III star $\xi$ Persei $^{\star}$
}

J. A. de Jong ${ }^{1,2}$, H. F. Henrichs ${ }^{1}$, L. Kaper ${ }^{1}$, J. S. Nichols ${ }^{3}$, K. Bjorkman ${ }^{4}$, D. A. Bohlender ${ }^{5}$, H. Cao ${ }^{6}$,

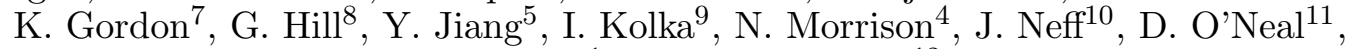
B. Scheers ${ }^{1}$, and J. H. Telting ${ }^{12}$

1 Astronomical Institute "Anton Pannekoek", University of Amsterdam, Kruislaan 403, 1098 SJ Amsterdam, The Netherlands; e-mail: huib@astro.uva.nl; lexk@astro.uva.nl

2 Leiden Observatory, University of Leiden, Niels Bohrweg 2, 2333 CA Leiden, The Netherlands e-mail: jdejong@strw.leidenuniv.nl

3 Harvard/Smithsonian Center for Astrophysics, 60 Garden Str., Cambridge, MA 02138, USA e-mail: jnichols@cfa.harvard.edu

4 Ritter Astrophysical Research Center, The University of Toledo, Toledo, OH 43606, USA e-mail: ndm@physics.utoledo.edu; karen@astro.utoledo.edu

${ }^{5}$ National Research Council of Canada, Herzberg Institute of Astrophysics, 5071 W. Saanich Road, Victoria BC, Canada V9E 2E7; e-mail: david.bohlender@nrc.ca

6 Beijing Astronomical Observatory, Beijing 100012, PR China; e-mail: caohl@class1.bao.ac.cn

7 Steward Observatory, University of Arizona, Tuczon, AZ 85712, USA; e-mail: kgordon@as.arizona.edu

8 Physics Dept. University of Montreal, PQ H3C 3J7 Montreal, Canada; e-mail: hill@astro.umontreal.ca

9 Tartu Observatory, 61602 Tõravere, Estonia; e-mail: indrek@aai.ee

10 Dept. of Physics and Astronomy, College of Charleston, Charleston, SC 29424, USA; e-mail: neffj@cofc.edu

11 Dept. of Astronomy and Astrophysics, Pennsylvania State University, University Park, PA 16802, USA e-mail: doneal@astro.psu.edu

12 Isaac Newton Group of Telescopes, NWO (Netherlands Organisation for Scientific Research), Apartado 321, 38700 Santa Cruz de La Palma, Spain; e-mail: jht@ing.iac.es

Received 17 November 2000 / Accepted 11 December 2000

Abstract. We present the results of an extensive observing campaign on the O7.5 III star $\xi$ Persei. The UV observations were obtained with the International Ultraviolet Explorer. $\xi$ Per was monitored continuously in October 1994 during 10 days at ultraviolet and visual wavelengths. The ground-based optical observations include magnetic field measurements, $\mathrm{H} \alpha$ and He I $\lambda 6678$ spectra, and were partially covered by photometry and polarimetry. We describe a method to automatically remove the variable contamination of telluric lines in the groundbased spectra. The aim of this campaign was to search for the origin of the cyclical wind variability in this star. We determined a very accurate period of 2.086(2) d in the resonance lines of Si IV and in the subordinate N IV and $\mathrm{H} \alpha$ line profiles. The epochs of maximum absorption in the UV resonance lines due to discrete absorption components (DACs) coincide in phase with the maxima in blue-shifted $\mathrm{H} \alpha$ absorption. This implies that the periodic variability originates close to the stellar surface. The phase-velocity relation shows a maximum at $-1400 \mathrm{~km} \mathrm{~s}^{-1}$ The general trend of these observations can be well explained by the corotating interaction region (CIR) model. In this model the wind is perturbed by one or more fixed patches on the stellar surface, which are most probably due to small magnetic field structures. Our magnetic field measurements gave, however, only a null-detection with a $1 \sigma$ errorbar of $70 \mathrm{G}$ in the longitudinal component. Some observations are more difficult to fit into this picture. The 2-day period is not detected in the photospheric/transition region line He I $\lambda 6678$. The dynamic spectrum of this line shows a pattern indicating the presence of non-radial pulsation, consistent with the previously reported period of $3.5 \mathrm{~h}$. The edge variability around $-2300 \mathrm{~km} \mathrm{~s}^{-1}$ in the saturated wind lines of C IV and N V is nearly identical to the edge variability in the unsaturated Si IV line, supporting the view that this type of variability is also due to the moving DACs. A detailed analysis using Fourier reconstructions reveals that each DAC actually consists of 2 different components: a "fast" and a "slow" one which merge at higher velocities.

Key words. stars - early-type - atmospheres - winds, outflows - magnetic fields

Send offprint requests to: J. A. de Jong,

e-mail: jdj@astro.uva.nl

* Based on observations obtained using the International

Ultraviolet Explorer, collected at NASA Goddard Space Flight

Center and Villafranca Satellite Tracking Station of the European Space Agency. 


\section{Introduction}

All $\mathrm{O}$ and many B stars show cyclical variability in their stellar winds. The most prominent features are the discrete absorption components (DACs) observed in UV P Cygni profiles. They accelerate through the blue-shifted absorption trough in a few days until they approach the terminal wind velocity $\left(v_{\infty}\right)$. The saturated profiles, in which DACs cannot be observed, show regular shifts in their blue edges, up to $10 \%$ in velocity. A key observation is that DACs appear periodically (Henrichs et al. 1988; Prinja 1988), and that their recurrence timescale is shorter in stars with a higher (projected) rotational velocity. This strongly suggests that stellar rotation determines the timescale of variability in OB-star winds. For a more extensive introduction and various examples of DAC behavior the reader is referred to Kaper et al. (1996).

The main unsolved issue is where the DACs originate. A comparison of different time series (see Kaper et al. 1999a) of DACs in the Si IV profile shows that for a given star the same recurrence timescale is observed over many years, but detailed changes occur from year to year.

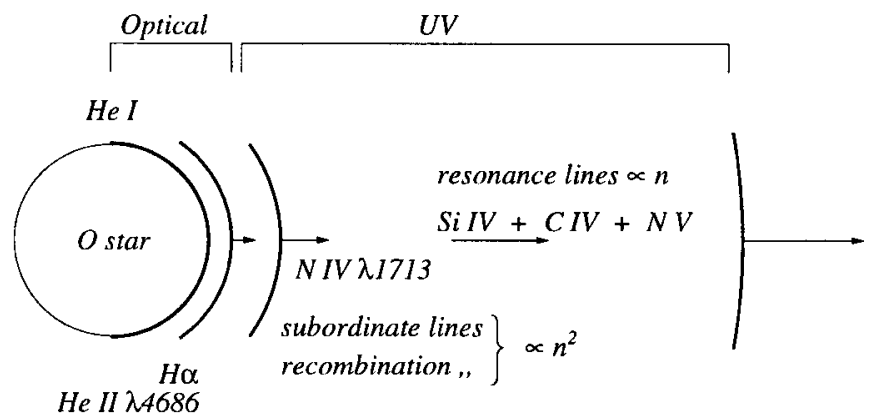

Fig. 1. Sketch of the line-forming regions probed in multiwavelength campaigns of $\mathrm{O}$ stars

The wind structures can be traced back to very low velocities: basically down to the $v \sin i$ value of the star (see for $\xi$ Per Henrichs et al. 1994). Together with the observed rotational modulation this argues in favor of a model invoking corotating structures which originate at or near the surface of the star. Such models have been proposed by several authors. Underhill \& Fahey (1984) investigated the possible effects of magnetic surface structures on O star winds. Mullan $(1984,1986)$ proposed that DACs are caused by CIRs in analogy with the solar wind. On the basis of the CIR model Cranmer \& Owocki (1996) performed radiative hydrodynamical calculations to investigate the impact of bright or dark spots at the surface of a rotating star on the dynamical structure of the stellar wind. The higher (or lower) flux from such a spot causes a local change in the radial flow properties, for example a higher density and a smaller outflow velocity. Due to the stellar rotation the perturbed stream is curved, so that further-out in the wind, the slow (or fast) stream interacts with the ambient flow resulting in a spiral shaped shock-front that corotates with the star. The physical origin of such a spot is not specified. One could think of the changes in surface temperature and velocity associated with non-radial pulsations (NRP) or a local increase in mass-loss rate due to the presence of a surface magnetic field. Cranmer \& Owocki showed that the resulting wind structure gives rise to features resembling DACs in UV resonance lines.

Figure 1 symbolically maps out the regions where the most important lines are formed in O-star atmosphere and wind. The strength of the UV resonance lines is proportional to (density $)^{1}$; therefore, they probe the wind over a much larger extent than e.g. $\mathrm{H} \alpha$ and the subordinate N IV $\lambda 1718$, which' strength falls off as (density) ${ }^{2}$; the latter, therefore, preferentially sample the innermost part of the wind. The deep photospheric lines are used to study pulsation behavior by means of Doppler imaging techniques. The timescales of pulsation, rotation and wind flow are all on the order of a day, which makes it particularly difficult to disentangle these effects, and a global network of observatories is needed to obtain the required time sampling.

We have conducted several multisite campaigns to simultaneously probe the stellar wind, its base and the underlying photosphere. Here we present the results obtained in the October 1994 campaign on one of the best studied O stars so far: $\xi$ Per O7.5 III(n)((f)). Its visual brightness $(V=4.0)$ and the short timescale of the wind variability (Prinja et al. 1987) make this star an excellent target for a detailed study.

\section{Observations and data reduction}

A schematic overview of the campaign is presented in Fig. 2. Over a period of about 10 days we collected 70 ultraviolet spectra with the International Ultraviolet Explorer (IUE), covering 1150-1900 A. Six average magnetic field values, each consisting of 14 measurements, were obtained with the University of Western Ontario (UWO) polarimeter attached to the CFHT (see Table 2 for the meaning of the acronyms used throughout this paper). High quality $\mathrm{H} \alpha$ spectra were collected from various longitudes around the globe: Canada (DAO, Victoria), USA (Ritter Observatory, Toledo, and BMO, Pennsylvania), Europe (OHP, France and JKT, Canary Islands), and China (BAO). In total 368 usable spectra were obtained, with an average time coverage of $70 \%$ per day during the 10-day campaign. The spectra from JKT, OHP and BMO also contain the weak He I $\lambda 6678$ absorption line. Average sampling times were 3 hours for the UV spectra and half an hour for the optical spectra. Spectropolarimetry was conducted at Pine Bluff Observatory (Wisconsin, USA, observer Bjorkman), but only one spectrum could be obtained. In this section we describe the basic data reduction procedures. Further processing for ensuring a homogeneous dataset is described in Sect. 3.1. 
Overview of $\xi$ Per Campaign October 1994 Date (UT)

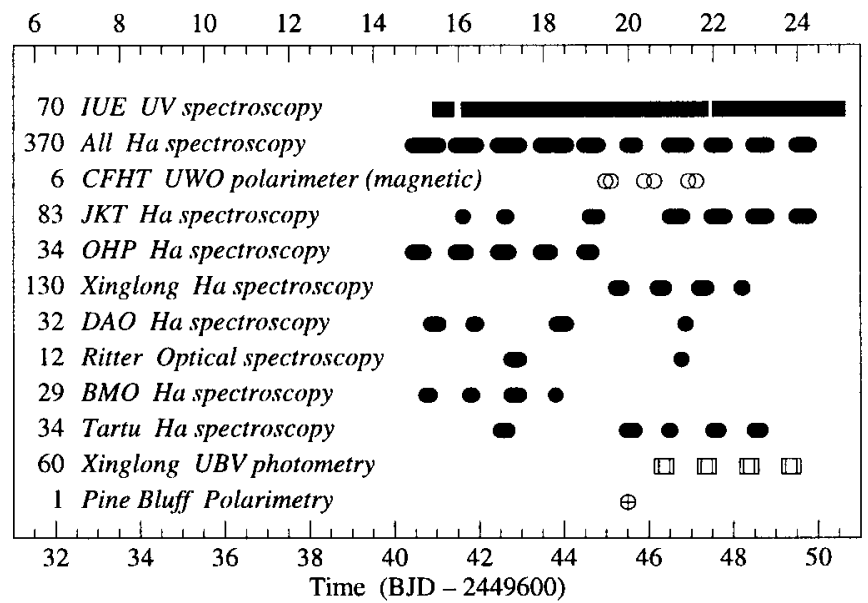

Fig. 2. Schematic overview of the October 1994 campaign on $\xi$ Per. The number of observations for each type of observation is indicated in the leftmost column

\subsection{Ultraviolet spectroscopy}

High-dispersion ultraviolet spectra $(R \simeq 10000)$ were obtained with the Short Wavelength Prime (SWP) camera on board the IUE satellite (Nichols, Henrichs, Scheers). The log of these observations is presented in Table 1. For a detailed description of the data reduction process we refer to Kaper et al. (1996). We used the Starlink IUEDR software package (Giddings 1981). Interstellar lines were used to optimize the wavelength calibration. The echelle-ripple correction was performed with the method described by Barker (1984). Reseau marks were removed by linear interpolation. The spectra were mapped on a uniform wavelength grid of $0.1 \AA$. The signal-to-noise ratio of the spectra is about 30 (see Henrichs et al. 1994).

\subsection{Magnetic field measurements}

Magnetic field measurements were obtained with the UWO photo-electric Poeckels cell polarimeter mounted at the Cassegrain focus of the $3.6 \mathrm{~m}$ Canada-France-Hawaii Telescope at Mauna Kea (observers Bohlender, Hill). With this instrument the Zeeman-splitting due to the longitudinal component of a magnetic field is measured in the $\mathrm{H} \beta$ line profile. The red and blue components can be discerned, because they are circularly polarized in opposite directions. The 2-channel photo-electric polarimeter uses two narrow-band ( $5 \AA$ ) filters to measure the red and blue wings of the $\mathrm{H} \beta$ line, each centered a few $\AA$ from the line center at the steepest gradient of the line to ensure the maximum signal (Landstreet 1982 and references therein).
Table 1. Journal of IUE observations of $\xi$ Per, October 1994. The ground station from which the satellite was controlled during the exposure is indicated in the last column (G1 = GSFC US1 shift, G2 = GSFC US2 shift, V = VILSPA)

\begin{tabular}{|c|c|c|c|c|c|c|}
\hline \# & $\begin{array}{l}\text { Image } \\
\text { SWP }\end{array}$ & $\begin{array}{c}\text { Day } \\
\text { Oct. } 1994\end{array}$ & $\begin{array}{c}\text { Start UT } \\
\text { h:min:s }\end{array}$ & $\underset{\mathrm{s}}{\operatorname{Expo} .}$ & $\begin{array}{c}\text { Mid Exp. } \\
\text { BJD-2449600 }\end{array}$ & \\
\hline 1 & 52410 & 15 & $13: 47: 09$ & 70 & 41.075 & $\mathrm{~V}$ \\
\hline 2 & 52413 & & $17: 01: 26$ & 70 & 41.210 & V \\
\hline 3 & 52419 & 16 & $05: 53: 43$ & 70 & 41.746 & G2 \\
\hline 4 & 52422 & & 08:40:31 & 70 & 41.862 & G2 \\
\hline 5 & 52426 & & $11: 49: 13$ & 70 & 41.993 & G2 \\
\hline 6 & 52430 & & $15: 54: 34$ & 70 & 42.163 & V \\
\hline 7 & 52434 & & $19: 26: 19$ & 70 & 42.310 & V \\
\hline 8 & 52437 & & $22: 25: 02$ & 75 & 42.434 & G1 \\
\hline 9 & 52441 & 17 & $01: 42: 32$ & 75 & 42.572 & G1 \\
\hline 10 & 52444 & & 04:54:01 & 75 & 42.705 & G1 \\
\hline 11 & 52448 & & $08: 08: 55$ & 75 & 42.840 & G2 \\
\hline 12 & 52451 & & $10: 27: 42$ & 75 & 42.936 & G2 \\
\hline 13 & 52455 & & $13: 59: 42$ & 75 & 43.084 & V \\
\hline 14 & 52458 & & $17: 15: 10$ & 75 & 43.219 & V \\
\hline 15 & 52463 & & $22: 23: 43$ & 75 & 43.434 & G1 \\
\hline 16 & 52466 & 18 & $00: 57: 32$ & 75 & 43.540 & G1 \\
\hline 17 & 52470 & & $04: 26: 05$ & 75 & 43.685 & G1 \\
\hline 18 & 52473 & & $07: 51: 40$ & 75 & 43.828 & G2 \\
\hline 19 & 52477 & & $10: 36: 23$ & 75 & 43.942 & G2 \\
\hline 20 & 52480 & & $12: 43: 39$ & 75 & 44.031 & G2 \\
\hline 21 & 52483 & & $15: 24: 40$ & 75 & 44.143 & V \\
\hline 22 & 52487 & & 20:05:59 & 75 & 44.338 & V \\
\hline 23 & 52490 & & $23: 33: 43$ & 75 & 44.482 & G1 \\
\hline 24 & 52494 & 19 & $02: 49: 11$ & 75 & 44.618 & G1 \\
\hline 25 & 52497 & & $06: 05: 30$ & 75 & 44.754 & G2 \\
\hline 26 & 52501 & & $09: 14: 44$ & 75 & 44.886 & G2 \\
\hline 27 & 52504 & & $11: 15: 41$ & 75 & 44.970 & G2 \\
\hline 28 & 52508 & & $14: 21: 44$ & 75 & 45.099 & $\mathrm{~V}$ \\
\hline 29 & 52511 & & $17: 09: 05$ & 75 & 45.215 & V \\
\hline 30 & 52515 & & $20: 40: 24$ & 75 & 45.362 & V \\
\hline 31 & 52517 & 20 & 01:10:08 & 75 & 45.549 & G1 \\
\hline 32 & 52520 & & 03:17:09 & 75 & 45.637 & G1 \\
\hline 33 & 52523 & & $05: 22: 17$ & 75 & 45.724 & G2 \\
\hline 34 & 52526 & & $07: 41: 49$ & 75 & 45.821 & G2 \\
\hline 35 & 52529 & & $09: 51: 35$ & 75 & 45.911 & G2 \\
\hline 36 & 52532 & & 12:03:04 & 75 & 46.003 & G2 \\
\hline 37 & 52535 & & $15: 46: 54$ & 75 & 46.158 & V \\
\hline 38 & 52539 & & 19:06:59 & 75 & 46.297 & V \\
\hline 39 & 52542 & & $21: 44: 19$ & 75 & 46.406 & G1 \\
\hline 40 & 52545 & 21 & $00: 12: 48$ & 75 & 46.509 & G1 \\
\hline 41 & 52548 & & $02: 28: 53$ & 75 & 46.604 & G1 \\
\hline 42 & 52551 & & 04:58:09 & 75 & 46.707 & G1 \\
\hline 43 & 52554 & & 07:08:29 & 75 & 46.798 & G2 \\
\hline 44 & 52557 & & $09: 27: 45$ & 75 & 46.895 & G2 \\
\hline 45 & 52560 & & $11: 54: 58$ & 75 & 46.997 & G2 \\
\hline 46 & 52563 & & $14: 39: 56$ & 75 & 47.111 & V \\
\hline 47 & 52566 & & $16: 53: 49$ & 75 & 47.204 & V \\
\hline 48 & 52571 & 22 & $03: 40: 20$ & 75 & 47.653 & G1 \\
\hline 49 & 52575 & & $06: 40: 30$ & 75 & 47.779 & G2 \\
\hline 50 & 52578 & & $08: 55: 41$ & 75 & 47.872 & G2 \\
\hline 51 & 52581 & & $11: 17: 39$ & 75 & 47.971 & G2 \\
\hline 52 & 52584 & & 13:50:08 & 75 & 48.077 & V \\
\hline 53 & 52588 & & $17: 14: 46$ & 75 & 48.219 & $\mathrm{~V}$ \\
\hline 54 & 52591 & & $19: 33: 51$ & 75 & 48.316 & V \\
\hline 55 & 52595 & & $22: 51: 57$ & 75 & 48.453 & G1 \\
\hline 56 & 52598 & 23 & 01:03:42 & 75 & 48.545 & G1 \\
\hline 57 & 52601 & & $03: 22: 27$ & 75 & 48.641 & G1 \\
\hline 58 & 52604 & & $05: 38: 14$ & 75 & 48.735 & G2 \\
\hline 59 & 52607 & & $07: 54: 57$ & 75 & 48.830 & G2 \\
\hline 60 & 52610 & & $10: 23: 40$ & 75 & 48.934 & G2 \\
\hline 61 & 52613 & & $12: 36: 57$ & 75 & 49.026 & G2 \\
\hline 62 & 52617 & & $15: 42: 34$ & 75 & 49.155 & V \\
\hline 63 & 52621 & & $19: 28: 23$ & 75 & 49.312 & V \\
\hline 64 & 52626 & 24 & $01: 57: 58$ & 75 & 49.582 & G1 \\
\hline 65 & 52629 & & $04: 16: 38$ & 75 & 49.679 & G1 \\
\hline 66 & 52633 & & $07: 20: 14$ & 75 & 49.806 & G2 \\
\hline 67 & 52636 & & $09: 37: 54$ & 75 & 49.902 & G2 \\
\hline 68 & 52639 & & $12: 04: 40$ & 75 & 50.004 & G2 \\
\hline 69 & 52649 & & $20: 27: 16$ & 75 & 50.353 & V \\
\hline 70 & 52652 & & $23: 10: 57$ & 75 & 50.466 & G1 \\
\hline
\end{tabular}

\subsection{Optical spectra}

Long-slit spectra centered on $\mathrm{H} \alpha$ were obtained at DAO, JKT, OHP, TO, and XO whereas échelle spectra were obtained at BMO and RO. The ESO MIDAS package was

used for the reduction of BMO, DAO, OHP and XO data. The spectra from JKT were reduced using NOAO's IRAF package. A large effort went into the reduction of the data 
Table 2. Description of the used instruments. The acronyms in the second column are used throughout this paper

\begin{tabular}{|c|c|c|c|c|c|c|}
\hline Observatory & & Telescope & Instrument & $R$ & $\lambda$ range $(\AA)$ & $N_{\text {spec }}$ \\
\hline Observatoire de Haute-Provence & $\mathrm{OHP}$ & $1.52 \mathrm{~m}$ & Aurélie & 50000 & 6500 to 6620 & 34 \\
\hline $\begin{array}{l}\text { Observatorio del Roque de los } \\
\text { Muchachos on La Palma, ING } \\
\text { Jacobus Kapteyn Telescope }\end{array}$ & JKT & $1.0 \mathrm{~m}$ & long-slit & 10000 & 6450 to 6650 & 83 \\
\hline Black Moshannon Observatory & $\mathrm{BMO}$ & $1.6 \mathrm{~m}$ & $\begin{array}{l}\text { fiber-fed } \\
\text { echelle }\end{array}$ & 10000 & 4780 to 8990 & 29 \\
\hline Astrophysical & $\mathrm{DAO}$ & $1.2 \mathrm{~m}$ & long-slit & 23000 & 6350 to 6850 & 32 \\
\hline Ritter Observatory & $\mathrm{RO}$ & $1.1 \mathrm{~m}$ & $\begin{array}{l}\text { fiber-fed } \\
\text { échelle }\end{array}$ & 26000 & 5495 to 6760 & 12 \\
\hline Xinglong Observatory & $\mathrm{XO}$ & $2.2 \mathrm{~m}$ & long-slit & 2500 & 5691 to 6928 & 130 \\
\hline Tartu Observatory & TO & $1.5 \mathrm{~m}$ & long-slit & 16000 & 6520 to 6600 & 34 \\
\hline
\end{tabular}

to ensure sufficient homogeneity for an accurate analysis. We describe for each observatory the specific reduction strategy, methods and problems.

\section{(i) Observatoire de Haute Provence}

$\mathrm{H} \alpha$ time series were obtained at $\mathrm{OHP}$ (observer Kaper) at the coudé focus of the $1.52 \mathrm{~m}$ telescope. We used the Aurélie spectrograph with the $2 \times 2048$ Thomson CCD detector and grating \#7 to obtain high-resolution spectra $(R=70000)$ in a wavelength region from 6500 to $6620 \AA$. During all runs, calibration frames were obtained regularly in order to correct for the bias and dark current levels of the CCD. Th-Ar arc spectra and tungsten flat-field exposures taken at $\sim 2$ hour intervals through the night provided wavelength calibration and correction for pixel-to-pixel variations of the detector, respectively. The exposure times were about $10 \mathrm{~min}$ in which a $S / N$ of 300 was obtained.

\section{(ii) ING La Palma, JKT}

$\mathrm{H} \alpha$ and He I $\lambda 6678$ spectra were obtained using the RBS spectrograph and a $1024 \times 1024$ TEK CCD on the $1.0 \mathrm{~m}$ Jacobus Kapteijn Telescope at the Roque de los Muchachos observatory on La Palma (observer Telting). We used the 2400 grating with a resolution of 10000 covering the wavelength region from 6450 to $6650 \AA$. $\mathrm{Cu}-\mathrm{Ne}$ calibration frames were taken after every movement of the telescope, since the spectrograph is attached to it. Dome and internal flatfields taken at the beginning and end of the night were used for correcting the pixel-to-pixel variations of the detector. A $S / N$ of 300 was achieved in exposure times of $5 \mathrm{~min}$.

\section{(iii) Black Moshannon Observatory}

The spectra were obtained with the $1.6 \mathrm{~m}$ telescope of BMO (State College, Pennsylvania) using a fiber-fed echelle spectrograph and a Texas Instruments $432 \times 808$ CCD with $15 \mu$ pixel size (observers Neff and O'Neal). They contain 15 orders covering a total wavelength range from 4780 to $8990 \AA$, except for a number of gaps between the orders. We first subtracted an average bias levelfrom each frame. Secondly, the positions of the orders were located using the Hough method (see Ballester 1994). The background was fitted by means of a $2 \mathrm{D}$ polynomial fit over the inter-order space and subtracted.

The orders were extracted using the optimal extraction method (see Horne 1986). The orders were flatfield corrected after the extraction procedure. We did not apply flatfielding to the original frames because the orders were too narrow to remove the intrinsic light variations in the original flat fields. The wavelength calibration was done by means of a $2 \mathrm{D}$ polynomial fit on the positions of the Th-Ar lines which were constrained by the echelle relation between the absolute order number and the central wavelength. $\mathrm{H} \alpha$ lies close to an order edge which made the normalization of this line rather difficult.

\section{(iv) Dominion Astrophysical Observatory}

At DAO (observer Jiang) we obtained long-slit spectra ranging from 6350 to $6850 \AA$ with the spectrograph including a Reticon detector attached to the $1.2 \mathrm{~m}$ McKellar telescope in the coudé focus. The dispersion was $10 \AA \mathrm{mm}^{-1}$. The spectra contain 20 rows of starlight and no background. We had to correct the flat fields for non-uniform illumination by smoothing them row by row over 250 pixels in the direction of the dispersion. Secondly, we divided the flat fields by the smoothed versions, which resulted in frames containing only pixel to pixel variations. We averaged the bias frames per night and performed the standard bias and flat-field correction. The final spectra were obtained with the optimal extraction method and with a wavelength calibration using Th-Ar spectra.

\section{$(v)$ Xinglong Observatory}

At XO (observer Cao) we obtained low dispersion $(R=$ 2500) long-slit spectra ranging from 5691 to $6928 \AA$ with the Cassegrain spectrograph attached to the $2.16 \mathrm{~m}$ telescope, using the maximum available dispersion of $50 \AA \mathrm{mm}^{-1}$. The spectra consist of 70 rows of starlight and sky background. The rest of the CCD below and above the stellar slit was exposed by a Ne lamp for wavelength calibration. Per night all bias frames and flat-field frames were averaged. A bias frame was subtracted from 
the raw science frames and flatfields. Subsequently, the science frames were divided by appropiate flatfields.

The Ne-lamp was only used at beginning and the end of the night, so we could not use them to correct for the wavelength changes during the night. Furthermore, they were taken through a different slit which may have caused additional errors in the line positions. The spectra indeed shift in wavelength during the nights. We used a diffuse interstellar band (DIB) at $6612 \AA$ to determine and correct for the shift of each spectrum.

\section{(vi) Ritter Observatory, Toledo USA}

The $1 \mathrm{~m}$ Ritter telescope (observers Gordon and Morrison) has a fiber-fed echelle spectrograph with a maximum resolution of 26000 . The detector was an EEV CCD chip with $1200 \times 800$ pixels of $22.5 \mu$ size.

The reduction of the data was done in the Interactive Data Language (IDL) with a specialized program written for Ritter Observatory data (Gordon 1995) based on methods detailed in Hall et al. (1994). The average bias and flat field were constructed on a pixel-by-pixel basis allowing the removal of cosmic-ray hits. The average bias was subtracted from the average flat field-, object-, and wavelength calibration frames. The flat field was used to determine the order and background templates. The background template was used to remove the scattered light from the frames after fitting a polynomial to the interorder background on a column by column basis. Cosmic-ray hits in the object- and wavelength calibration frames were removed by comparing them with the average flat field. The orders were extracted using a profile-weighted extraction method. The wavelength calibration was accomplished by scaling all the comparison lines to one order and fitting a polynomial to the result, iteratively removing points until a preset standard deviation was achieved.

\section{(vii) Tartu Observatory}

At TO in Estonia (observer Kolka) we obtained 34 intermediate resolution $(R=16000) \mathrm{H} \alpha$ spectra using a long-slit Cassegrain spectrograph mounted on the $1.5 \mathrm{~m}$ telescope. A ST-6 CCD camera was used and Ne arc spectra were taken for the wavelength calibration. In all cases the exposure time was 10 min, yielding a typical $S / N$ of $60-80$. In order to increase the $S / N$ to match the other observatories we averaged blocks of spectra within time intervals of one hour (24 averaged spectra). The wavelength calibration was very problematic, because only two $\mathrm{Ne}$ lines fell within the short wavelength range of the detector. An attempt to find the non-linear dispersion coefficients was made by comparing the $\xi$ Per spectra with simultaneously taken spectra from other observatories, but no reliable match could be achieved, and consequently we could not use these spectra for the time series analysis.

\subsection{Removal of telluric lines}

Many of the $\mathrm{H} \alpha$ line profiles are heavily contaminated with about 20 distinct telluric lines. This contamination varies from night to night, mainly as a function of the amount of water vapor in the lower atmosphere. To remove these effects we first created a list of the telluric lines from a spectrum of a star with very broad lines (observed within the same night) and constructed a template telluric spectrum, which can be scaled as a function of average line strength and line width, and shifted in wavelength. In an automatic fitting procedure the contaminated source spectrum is divided by such a template and the three parameters are adjusted until the telluric lines are removed. Here follows a description of the method.

1. The telluric lines were identified by eye in the spectral region around the $\mathrm{H} \alpha$ profile of the rapidly rotating standard star $\zeta$ Aql, A0V. A spline curve was fitted through selected uncontaminated points and the stellar spectrum was divided out, which leaves a spectrum $S(\lambda)$ of only telluric lines. This spectrum was inverted $\left(S^{\prime}(\lambda)=1-S(\lambda)\right)$ and modeled using a multiple Gaussian function:

$$
S^{\prime}(\lambda)=\sum_{i=1}^{N} A_{i} \exp \left[-\left(\frac{\lambda-\lambda_{0 i}}{w_{i}}\right)^{2}\right]
$$

Here $N$ is the total number of telluric lines. In order to obtain the line depths $A_{i}$, central wavelengths $\lambda_{0 i}$ and line widths $w_{i}$ we fitted to each line a single component of Eq. (1), or with up to 5 components in case of blended lines. The set of parameters $\left(A_{i}, w_{i}, \lambda_{0 i}\right)$ defines a template telluric spectrum;

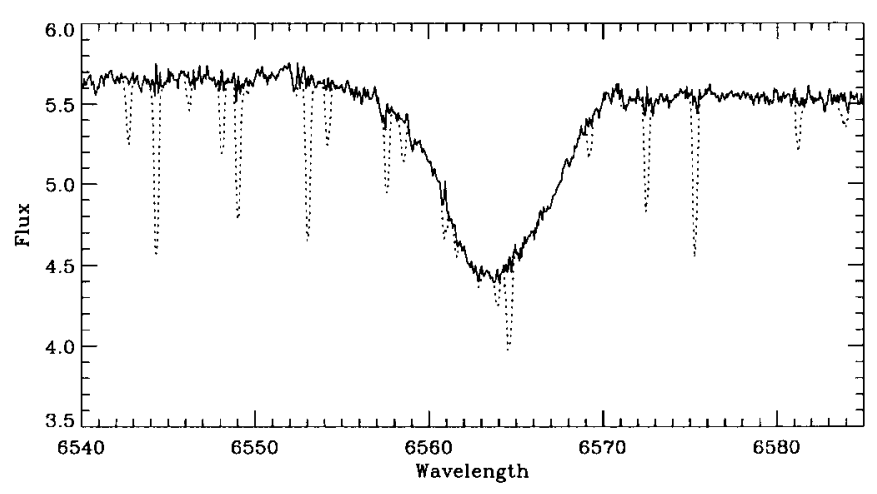

Fig. 3. An $\mathrm{H} \alpha$ spectrum of $\mathrm{RO}$ is shown before and after the removal of the telluric lines. The raw spectrum is shown as a dotted line

2. Given a template, relative optical depth $\Delta \tau$, relative line width $\Delta w$ and wavelength shift $\Delta \lambda$ a particular telluric spectrum $T(\lambda)$ is constructed as follows:

$$
\begin{aligned}
& T(\lambda)=\sum_{i=1}^{N}\left(1-\mathrm{e}^{-\tau_{i} \Delta \tau}\right) \mathcal{G}(\lambda) \\
& \mathcal{G}(\lambda)=\exp \left[-\left(\frac{\lambda-\lambda_{0 i}-\Delta \lambda}{w_{i} \Delta w}\right)^{2}\right] \\
& \tau_{i}=-\ln \left(1-A_{i}\right) .
\end{aligned}
$$




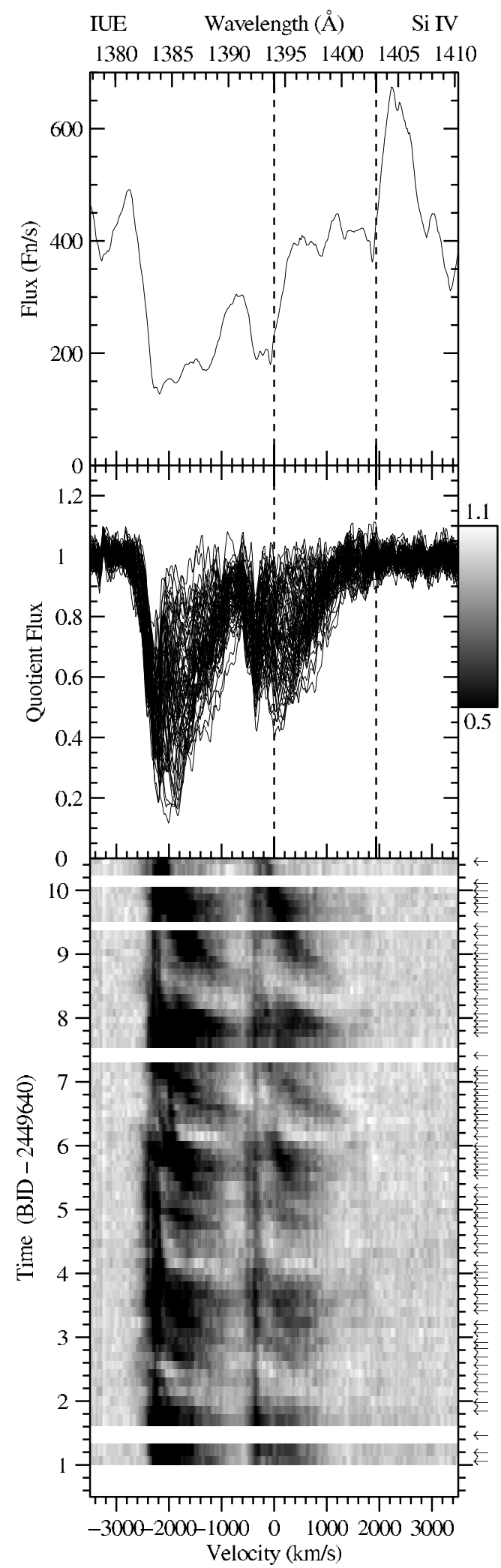

گेPer O7.5 III (n)((f)) $15-24$ October 1994 IUE Wavelength $(\AA) \quad$ N IV

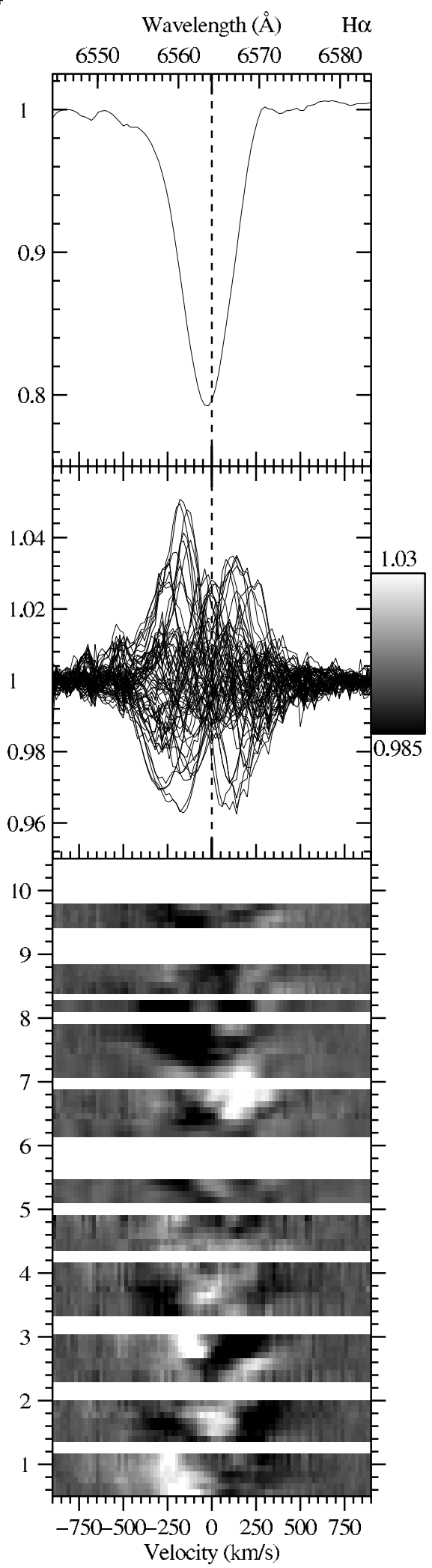

Fig. 4. Time series of the Si IV resonance doublet and the subordinate N IV and H $\alpha$ lines of the O7.5 III star $\xi$ Per in October 1994. Time is running upwards. Intensity is represented in levels of grey. To enhance the contrast the shown spectra are divided by templates (see Sect. 5.3 for an explanation of the UV templates), which are shown in the upper panels. The Si IV line shows a regular pattern of recurring DACs (period about $2 \mathrm{~d}$ ) with detailed changes from event to event. A clear correlation can be seen between these DAC events and the absorption in N IV and $\mathrm{H} \alpha$. It is also clear that the $\mathrm{H} \alpha$ variations are much more complicated 
3. A region containing strong telluric lines is chosen in the contaminated source spectrum $C(\lambda)$. In this region a polynomial $P(\lambda)$ is fitted to the continuum, which is compared to a residual spectrum $R(\lambda)=$ $C(\lambda) /(1-T(\lambda, \Delta \tau, \Delta w, \Delta \lambda))$. When the residual contains the least amount of telluric lines the following $\chi^{2}$ should be minimal:

$$
\chi^{2}=\sum_{i}\left(R\left(\lambda_{i}, \Delta \tau, \Delta w, \Delta \lambda\right)-P\left(\lambda_{i}\right)\right)^{2} .
$$

4. We adjusted $\Delta \tau, \Delta w$ and $\Delta \lambda$ and iterated from step 2 until a minimal $\chi^{2}$ was achieved using the Fletcher-Reeves-Polak-Ribiere minimization algorithm (see Press et al. 1992). However, before this fitting procedure could be started, we first shifted the template spectra for a specified range of $\Delta \lambda$ values, until an approximate optimal value for $\Delta \lambda$ was reached.

It was sufficient to make a line list from only a single $\zeta$ Aql spectrum of OHP (highest spectral resolution) in order to remove the telluric lines completely from all spectra regardless of observatory or time (see Fig. 3 for an example).

\section{Combination of the datasets}

\subsection{Creation of a homogeneous dataset}

For each observatory the spectra were normalized, transposed to the stellar rest frame, converted to velocity and rebinned to the same grid. The normalization of the UV lines was done with a straight-line fit through the continuum around each studied line individually. The continua of the optical spectra were much more curved and often contained spurious features. Therefore, we normalized them by fitting $3^{\text {rd }}$ order polynomials to a sufficiently large range of carefully selected continuum points. In the case of $\mathrm{H} \alpha$ we removed the telluric lines before normalization, because their presence affects the determination of the continuum level. The used continuum regions are given in Table 3 . The normalization of the $\mathrm{BMO} \mathrm{H} \alpha$ spectra was quite problematic, because this line lies close to the edge of an order, leaving little continuum at one side. Also the XO spectra gave problems: because of the low resolution and a nearby diffuse interstellar band (DIB) there were very few continuum points close to the blue side of $\mathrm{H} \alpha$. In both cases this degraded the accuracy of the polynomial fits. The continuum around the He I $\lambda 6678$ line was so poorly defined that we divided the spectra by their time average before normalization. In this way many small-scale spectral features were removed, thereby making the normalization more accurate.

The selected lines were converted to velocity scale using the laboratory wavelengths in Table 3 . In the case of doublets we used the shorter wavelength of the two lines.

All optical spectra were rebinned to a resolution of $15000\left(20 \mathrm{~km} \mathrm{~s}^{-1}\right)$ which is somewhat higher than the resolution of the JKT spectra (10000) but lower than the resolution of the other spectra. We deconvolved the XO spectra by a Gaussian profile with a FWHM of $\sqrt{100^{2}-20^{2}} \mathrm{~km} \mathrm{~s}^{-1}$ to increase their resolution artificially to 15000 .

Finally, we divided the individual spectra by their average (optical lines) or a least-absorption template (UV lines). Such a UV template is obtained by selecting statistically the highest flux points taking into account the noise (see Kaper et al. 1999a). We used the same template as described in Kaper et al. (1999a). The resulting dynamic quotient spectra of Si IV, N IV and H $\alpha$ are shown in Fig. 4. We define "dynamic spectra" as a series of spectra plotted as function of time.

\subsection{Comparison between different observatories}

We checked for possible systematic differences between spectra obtained at the different observatories by comparing $\mathrm{H} \alpha$ spectra taken at approximately the same time. We could only compare OHP with JKT, BMO with RO and BMO with DAO. In case of TO this comparison could not be applied, since the TO spectra were matched to simultaneously taken data (see Sect. 2.3).

From this comparison we found, unexpectedly, that the RO spectra were up to $3 \%$ deeper than the BMO spectra in the velocity range from $0-300 \mathrm{~km} \mathrm{~s}^{-1}$ whereas the BMO and DAO spectra agreed within the noise. This deviation of the RO spectra did practically not affect the period analysis (see Sect. 5.5), probably because these spectra were all taken within a single small time interval.

The XO spectra also seem to differ systematically, although we could only check this by comparing their average with the average JKT spectrum. For XO the average spectrum is about $2.5 \%$ less deep in the wings. The same JKT spectra agree within the $S / N$ to the simultaneously taken $\mathrm{OHP}$ spectra. We were not able to find an explanation for this effect, but we note that the deviations were less severe in spectra of another star (19 Cep), which do not show strong variations on a time scale of one day. However, the period analysis showed that including the $\mathrm{XO}$ data causes a strong 1-day signal which was not seen in the analysis of any other combination of observatories. We therefore decided to correct for the deviation in the $\mathrm{XO}$ data using a spline fit through the quotient of the average JKT and average XO spectra.

We could not include the TO spectra in our further analysis. Even though the wavelength calibrations were corrected using simultaneously taken spectra from other observatories, equivalent-width comparisons showed unexplained differences, which strongly affected the results of the period analysis.

\section{Overview of the observed line profile variations}

Figure 4 shows dynamic quotient spectra of the Si IV, N IV and $\mathrm{H} \alpha$ lines transposed to the stellar rest frame. For the construction of the quotient $\mathrm{H} \alpha$ spectra we used a plain average profile. In this figure, the $\mathrm{H} \alpha$ spectra were 
Table 3. List of optical and UV lines studied in this paper. The third and fourth columns list the rest wavelengths used to convert to radial velocity units. Furthermore, we show the regions which were excluded in the continuum fitting for normalization (see Sect. the order of the polynomial used for the normalization of the continuum

\begin{tabular}{lllllll}
\hline Line & Type of line & $\lambda_{0}(1)(\AA)$ & $\lambda_{0}(2)(\AA)$ & $\begin{array}{l}\text { region excluded in } \\
\text { normalization }\left(\mathrm{km} \mathrm{s}^{-1}\right)\end{array}$ & $\begin{array}{l}\text { polynomial } \\
\text { order }\end{array}$ & $\begin{array}{l}\text { Formation } \\
\text { region }\end{array}$ \\
\hline $\mathrm{N} \mathrm{V}$ & resonance & 1238.821 & 1242.804 & $<3046$ & $0^{*}$ & wind \\
$\mathrm{Si} \mathrm{IV}$ & resonance & 1393.755 & 1402.770 & -3000 to 3000 & 1 & wind \\
$\mathrm{N}$ IV & sub-ordinate & 1718.550 & & -3000 to 3000 & 1 & inner wind \\
$\mathrm{C} \mathrm{IV}$ & resonance & 1548.187 & 1550.772 & -3550 to 2360 & 1 & wind \\
$\mathrm{H} \alpha$ & recombination & 6562.817 & & -1000 to 1000 & 3 & photosphere + inner wind \\
$\mathrm{He} \mathrm{I}^{* *}$ & & 6678.154 & $6683.209(\mathrm{He} \mathrm{II})$ & -500 to 750 & 3 & photosphere \\
\hline
\end{tabular}

* Only the red side of this line showed a proper continuum.

** Only present in JKT, BMO, DAO and XO spectra.

smoothed by taking a running mean with a time sampling of about 2 hours, where the contributing spectra were weighted according to their epoch within the sampling width. The resulting quotient spectra have a $S / N$ of better than 200. The development of five strong Si IV DAC events can be seen; in most cases the events consist of different components with different kinematic properties. The five strongest DAC events also clearly show up in the low-velocity region of the N IV lines, down to $-200 \mathrm{~km} \mathrm{~s}^{-1}$, i.e. the $v \sin i$ value of the star. They recur every 2 days. The same holds for the $\mathrm{H} \alpha$ region: the strongest absorption around $-250 \mathrm{~km} \mathrm{~s}^{-1}$ coincides with the epoch of enhanced absorption in N IV at intermediate velocities, and the appearance of a DAC in SiIv around $-1500 \mathrm{~km} \mathrm{~s}^{-1}$. This unambiguously shows that the wind structures responsible for DACs can be traced down to the stellar surface and must have a large physical extent (several $R_{\star}$ ).

In Fig. 5 we show the normalized $\mathrm{H} \alpha$ line profiles in order to illustrate how these profiles change when the enhanced blue shifted absorption occurs. The maxima in equivalent width $(E W)$ measured between $[-500,-200] \mathrm{km} \mathrm{s}^{-1}$ (see Sect. 5.5.1) are indicated by the solid tickmarks (the dotted tickmarks in between indicate the $E W$ minima). It is clear that most variations occur in the blue wing where the profile is even visible up to $-600 \mathrm{~km} \mathrm{~s}^{-1}$ during the $E W$ maximum around $M B J D=8$. The central line depth and red wing remain relatively constant, with exceptions around $M B J D=2.6$ and $M B J D=6.8$ where some emission appears in the red wing.

Variations of a different type are also present in the data. In Fig. 6 the edge variability near $-2600 \mathrm{~km} \mathrm{~s}^{-1}$ of the saturated P Cygni profiles of CIV and NV is shown, compared with the Si IV edge. This variability is not periodic, but it is clear that the three edges move in concert. A relation with the DAC events is suggested when comparing to Fig. 9. Some DAC events reach a higher terminal velocity than others. An explanation might be that they have a different impact on the edge.
The photospheric He I $\lambda 6678$ line shows variability on a short timescale. In Fig. 7 narrow absorption features are visible (notably around $0.8,4.6$ and 8.6 $M B J D$ ), which correspond with spectral variations on a much shorter timescale than in all other lines. Our sampling rate is too low to determine a period for these variations, but we will show in Sect. 7.2 that they belong to non-radial pulsations (NRP).

\section{Quantitative analysis}

In the previous section we showed the characteristics of the cyclical wind variability. Now we will quantify this variability by means of equivalent width measurements, temporal variance spectra (TVS), DAC modeling and time series analysis.

\subsection{Equivalent Width measurements}

We measured the equivalent widths $(E W)$ in $\mathrm{H} \alpha$ and the UV resonance lines in velocity intervals where the appearance of DAC and edge variability is most prominent. Negative $E W$ means net emission. For the error computation we followed Chalabaev \& Maillard (1983) using the empirical noise model for IUE spectra according to Henrichs et al. (1994):

$\frac{F_{\lambda}}{\sigma_{\exp }}=29.8 \tanh \left(\frac{F_{\lambda}}{321}\right)$

where $F_{\lambda}$ is the flux (in units of flux density numbers), and $\sigma_{\text {exp }}$ is the expected noise. The results are shown in Fig. 8.

The shape of the $E W$ curve is very similar for both the edge and the blue-shifted absorption part of the line profiles of Si IV, N IV and C IV, though the $E W$ changes in the edge and those at lower velocities are different in character. The pattern of slow increase and sudden decrease in absorption repeats itself about every 2 days. This behavior is also found in the $\mathrm{H} \alpha$ line in the absorption region between -500 to $-200 \mathrm{~km} \mathrm{~s}^{-1}$, although the absorption maxima lag about 0.2 days in time compared to Si IV. However, the $E W$ of the $\mathrm{H} \alpha$ and $\mathrm{He}$ I lines between -200 


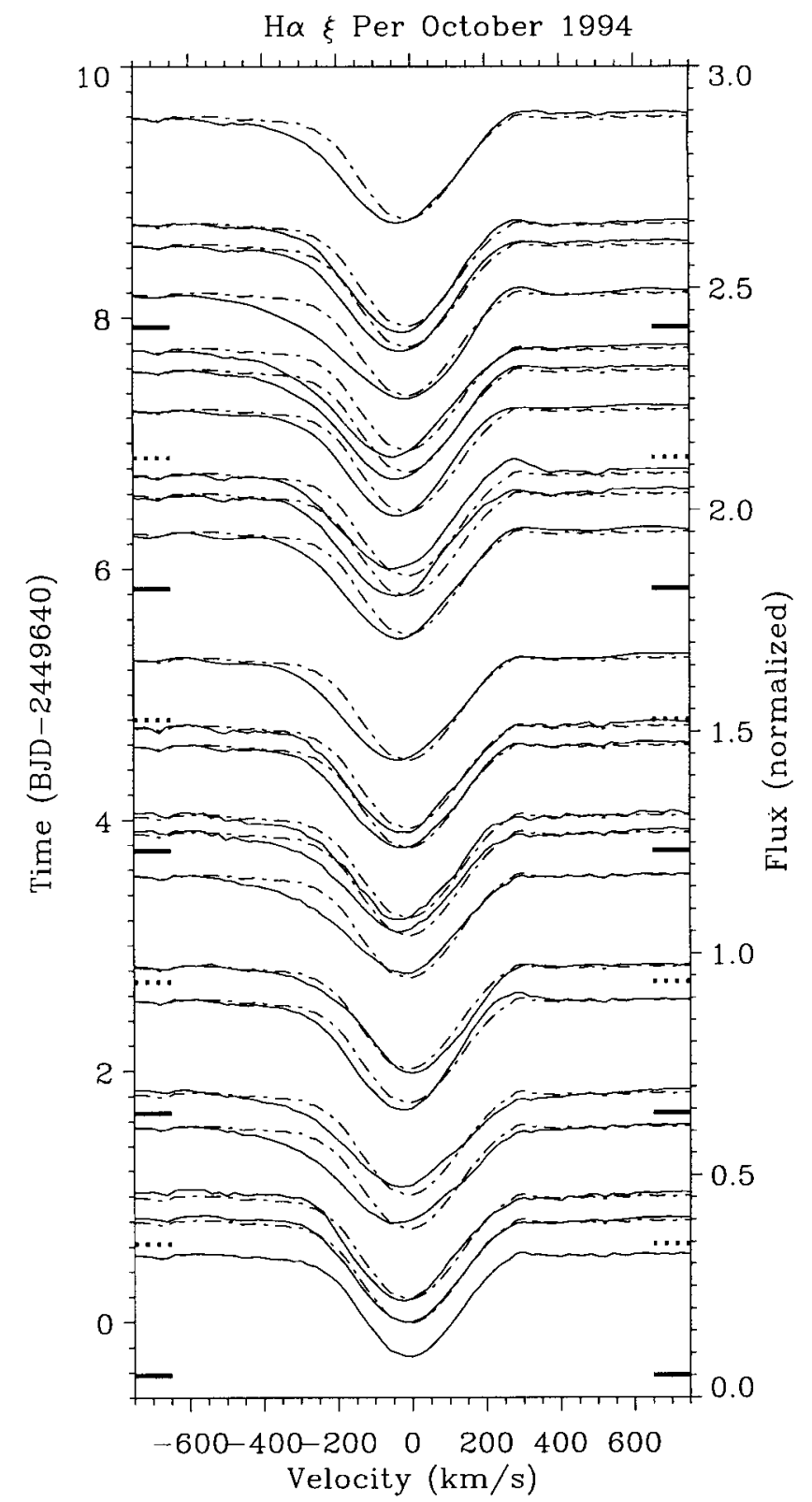

Fig. 5. Normalized $\mathrm{H} \alpha$ line profiles. Each line profile is an average of all profiles within a window of 0.25 days. The first profile is overplotted for comparison (dash-dotted lines). The vertical displacement of the line profiles is equal to the time interval between the observations. The scale on the right $y$-axis shows the lineflux with an arbitrary offset. Most of the variations are visible in the blue wing of the line. Note the enhanced blue-shifted absorption every two days

and $200 \mathrm{~km} \mathrm{~s}^{-1}$ show clearly a different pattern with a strong decrease in $E W$ around $M B J D=7$ just half a cycle before the strongest absorption at $M B J D=8$.

The EWs of the edge of SiIV, C IV and NV behave similarly, although there is no clear variation with a 2-day period. The $E W$ drops quite suddenly after $M B J D=6.5$. Note also that the lowest $E W$ in all edges coincides with the highest $E W$ in $\mathrm{H} \alpha$ at $M B J D=8$. If they are physically related this would be remarkable considering the entirely different wind regions which these lines should probe.
Table 4. CFHT longitudinal magnetic field results from $\mathrm{H} \beta$ polarimetry of $\xi$ Per. Each value is an average of 14 successive measurements

\begin{tabular}{crc}
\hline BJD-2449640 & $\begin{array}{r}B_{1} \\
\mathrm{G}\end{array}$ & $\begin{array}{c}\sigma\left(B_{\mathrm{l}}\right) \\
\mathrm{G}\end{array}$ \\
\hline 4.956 & 31 & 77 \\
5.093 & 136 & 82 \\
5.875 & -79 & 83 \\
6.117 & 60 & 69 \\
6.920 & 36 & 70 \\
7.109 & -13 & 93 \\
\hline
\end{tabular}

\subsection{Magnetic field analysis}

The longitudinal magnetic field strength is proportional to the differential polarization measured between the two wings of the line, which is in our case $\mathrm{H} \beta$ (cf. Mathys 1989). The derived values with their $1-\sigma$ error bars are listed in Table 4 and plotted in Fig. 8. Each quoted value is an average of 14 successive measurements. The average value of all measurements is $27 \pm 70 \mathrm{G}$, i.e. consistent with a non-detection. No periodicity or correlation is apparent. Although this is the most accurate search for a magnetic field in an O star to date, it is clear that the error bars on the magnetic field measurements are still too large to claim a significant field detection.

\subsection{DAC modeling}

We used a least-absorption template in order to isolate the migrating DACs from the underlying P Cygni profile. This template was derived using a rigorous statistical method which is described in detail in Kaper et al. (1999a). The N IV and SiIV quotient spectra shown in Fig. 4 were obtained with this template.

The DACs in the quotient spectra of Si IV are modeled using the method described by Henrichs et al. (1983, see also Telting \& Kaper 1994). The DACs are assumed to be plane-parallel slabs of material in the line of sight. They give rise to a Gaussian shaped absorption component at a velocity $v_{\mathrm{c}}$ with a broadening parameter $v_{\mathrm{t}}$ and central optical depth $\tau_{\mathrm{c}}$. The intensity of the component is described by:

$I(v)=\exp \left(-\tau_{\mathrm{c}} \exp \left[-\left(\frac{v-v_{\mathrm{c}}}{v_{\mathrm{t}}}\right)^{2}\right]\right)$.

We assume that the DACs can be modeled simultaneously using the doublet separation and the ratio of oscillator strengths of the doublet members (Table 3 ). The 3 free parameters are fitted for each pair of DACs by means of a $\chi^{2}$-method. In most cases a simultaneous fit with 3 or 4 DACs is needed. The column density $N_{\text {col }}$ is computed according to Henrichs et al. (1983):

$N_{\mathrm{col}}=\frac{m_{\mathrm{e}} c}{\pi e^{2}} \frac{\sqrt{\pi}}{f \lambda_{0}} \frac{\tau_{\mathrm{c}} v_{\mathrm{t}}}{\left(1+v_{\mathrm{c}} / c\right)}$

where $\pi e^{2} / m_{\mathrm{e}} c$ has its usual meaning, and $f$ is the oscillator strength of the transition at wavelength $\lambda_{0}$. The results 

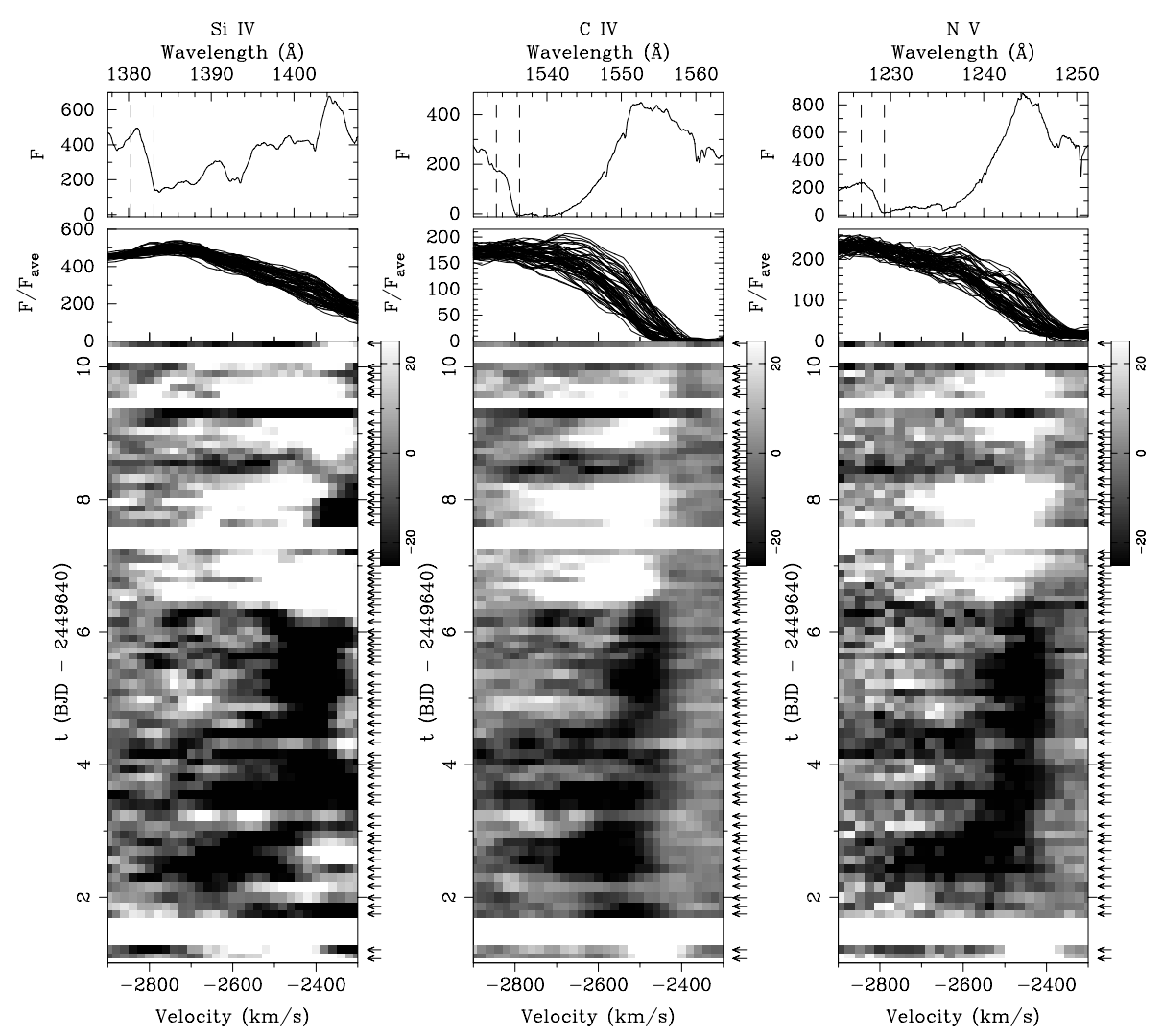

Fig. 6. Parallel edge variability in the UV resonance lines of Si IV, C IV and N V. The upper panels show the P Cygni profiles indicating the regions displayed below between the dashed lines. The middle panels show an overplot of the profiles. The bottom panels show a dynamic spectrum of the edge variability after subtraction of the mean profile. Darker areas correspond to relatively high edge velocities. Although the variations are similar in all three lines, no apparent periodicity can be observed

of the model fits are shown in Fig. 9. The evolution of the column density shows that a strong DAC appears every 2 days. Such DACs are always preceded by a weaker DAC, which peaks in $N_{\text {col }}$ about one day earlier. All DACs become detectable between -1000 and $-1500 \mathrm{~km} \mathrm{~s}^{-1}$ with a $v_{\mathrm{t}} \simeq 700 \mathrm{~km} \mathrm{~s}^{-1}$ and subsequently narrow as they move towards more negative velocities. The strong events coincide with the absorption features in N IV and the maximum $E W$ in the blue wing of $\mathrm{H} \alpha$. Their column densities alternate between about $410^{12} \mathrm{~cm}^{-2}$ and $610^{12} \mathrm{~cm}^{-2}$. The strong event around $M B J D=3.6$ is followed up by a weaker event around $M B J D=5.7$ and finally a stronger event appears around $M B J D=7.8$. We also see a persistent component which remains near the edge velocity between $M B J D=4.4$ and $M B J D=6$, which seems to be unrelated to any other event. An interesting event is the apparent crossing of two DACs around $M B J D=6.2$ (this also occured in other years, see Kaper et al. 1999a).

\subsection{Temporal variance spectra}

The amount of variability in the line profiles can be quantified by computing temporal variance spectra ( $T V S$, see
Fullerton et al. 1996). The $T V S$ at velocity bin $j$ is defined as follows:

$T V S_{j}=\frac{1}{N-1} \sum_{i=1}^{N}\left(\frac{F_{i j}-\bar{F}_{j}}{\sigma_{i j}}\right)^{2}$.

Here $F_{i j}$ and $\sigma_{i j}$ are the flux and the corresponding errors for wavelength bin $j$ in spectrum $i . \bar{F}_{j}$ is the average flux in bin $j$. The $\sigma_{i j}$ values of the UV lines were computed using Eq. (4).

For the optical lines we treated each observatory differently, because the dependence of $S / N$ on the flux is closely related to the type of spectrograph, quality of the calibration frames and the reduction method. In case of $\mathrm{H} \alpha$ the variability is quite large compared to the noise. We therefore only simply measured the noise in the continuum next to the profile assuming that it is proportional to $\sqrt{F}$. In contrast, the amplitude of the variability in the He I $\lambda 6678$ line is only slightly larger than the noise. For the noise measurements in this line we took into account the flux level of the unnormalized and in case of échelle spectra also unflatfielded spectra. We measured the $S / N$ in many continuum points and fitted a Poisson distribution function through these points $\left(F / \sigma_{\mathrm{F}}=\sqrt{g F}\right)$. Using these fits we determined the noise in each velocity bin of the normalized spectra. 

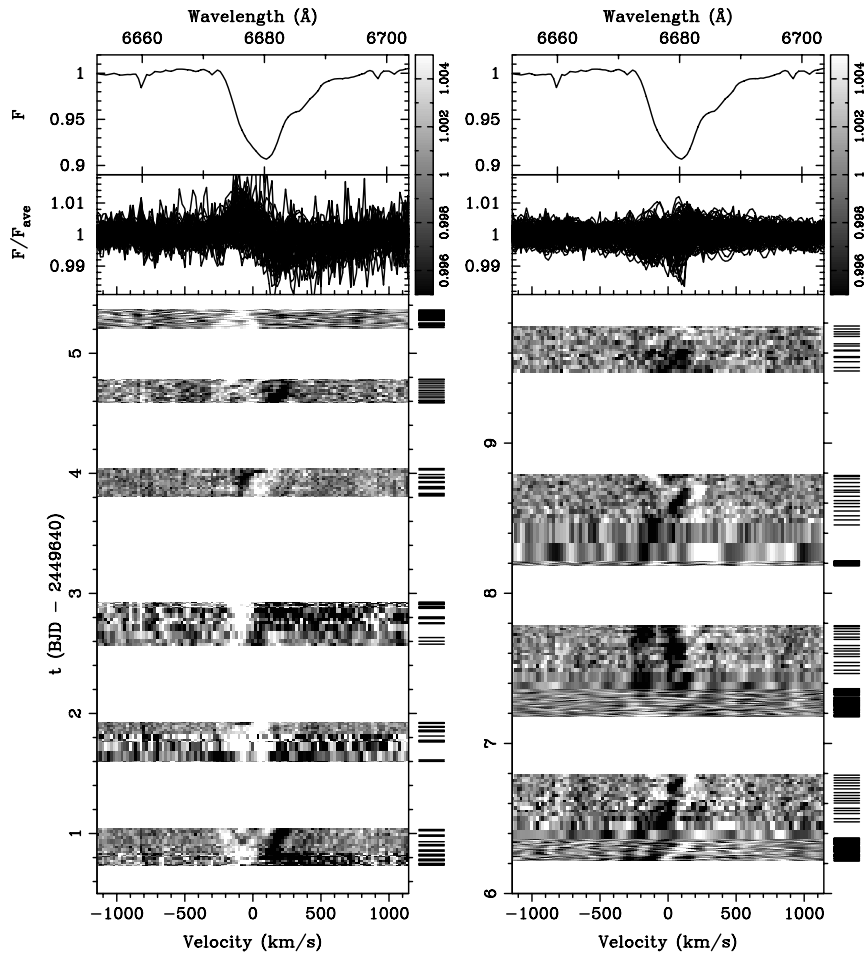

Fig. 7. Dynamic spectra of He I $\lambda 6678$ during the first part of the campaign (left) and second part (right). The red-ward moving patterns between $M B J D=6$ and $M B J D=9$ are most likely due to non-radial pulsations (see also Fig. 19)

The resulting TVS are shown in Fig. 10. This figure clearly shows that the different lines probe the variability in different regions. He I only shows variability within $\pm v \sin i$ with respect to the stellar rest frame, whereas the maximum amplitude of the variations move towards increasing negative velocity going from $\mathrm{H} \alpha, \mathrm{N}$ IV, Si IV, N V to C IV (the latter line is saturated at lower velocities).

\subsection{Period search}

We performed a standard CLEAN analysis (Roberts et al. 1987) on all UV and optical lines. In this method the discrete Fourier transformation (DFT) is deconvolved with the DFT of the window function, thereby removing the power which originates in the data sampling. This method gave the least amount of spurious peaks when applied to synthetic data with the same properties as our optical and UV data. Moreover, a comparison between different time series analysis methods by Carbonell et al. (1992) shows that the CLEAN method is most applicable to our randomly gapped and relatively short time series.

\subsubsection{Periods in EW measurements}

To enable a comparison with earlier measurements, our first period search was done on the $E W$ measurements (see Sect. 5.1 and Fig. 8). The resulting power diagrams (see Fig. 11) clearly show the same periodicity with a frequency of $0.48 \mathrm{c} / \mathrm{d}$ in all UV lines and $\mathrm{H} \alpha$.

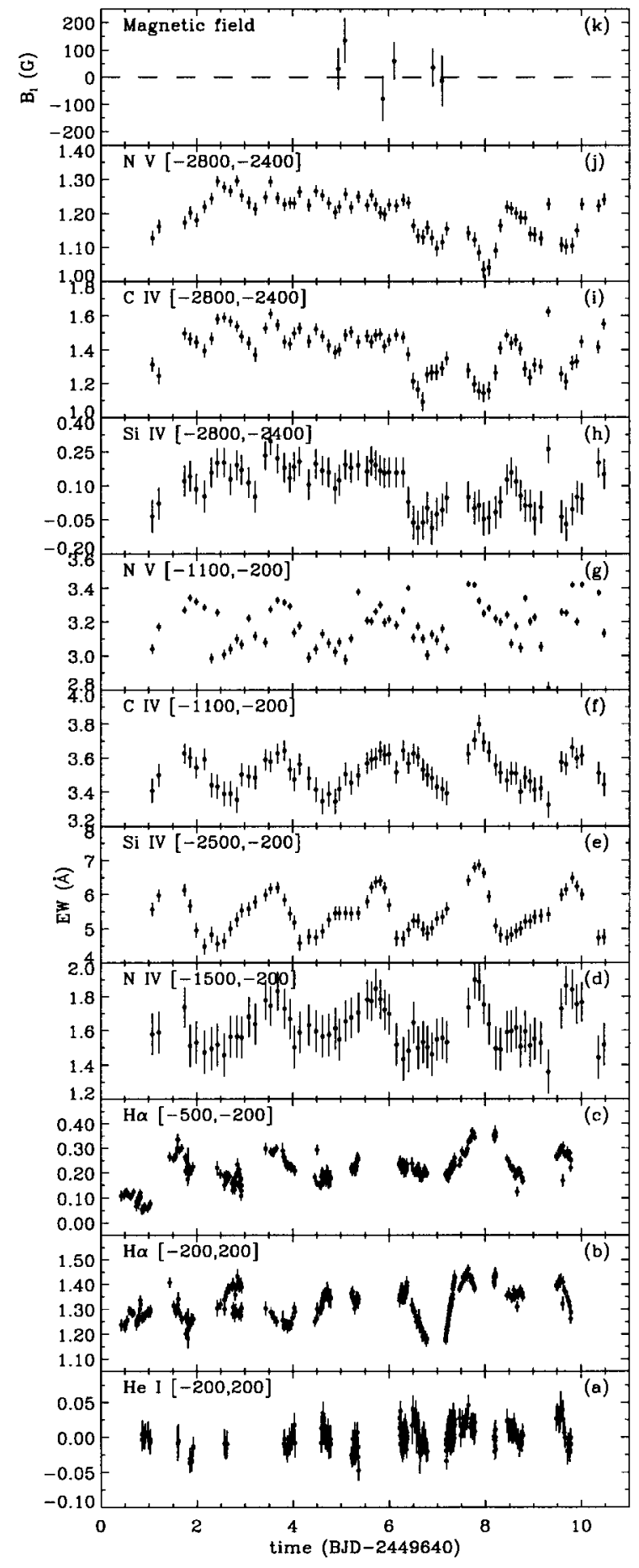

Fig. 8. Equivalent Width $(E W)$ measurements of all lines at different velocity intervals (a-j), and magnetic field measurements $\mathbf{k}$ ) as a function of time. We clearly see variations with a $2 \mathrm{~d}$ period in the velocity interval $[-200,-2500] \mathrm{km} \mathrm{s}^{-1}$ (c-g). The $E W$ of the edges of the UV resonance lines and the $\mathrm{H} \alpha$ and He I line between -200 and $200 \mathrm{~km} \mathrm{~s}^{-1}$ do not show such a clear periodicity. Furthermore, the $E W$ maxima of $\mathrm{H} \alpha$ lag about 0.2 days behind compared to those of Si IV

We determined the frequencies of maximum power $\left(f_{0}\right)$ by means of Gaussian fits. The errors were derived from the width and height of the peak and the noise level in the power diagram. The procedure is described in detail 
(a)

(b)

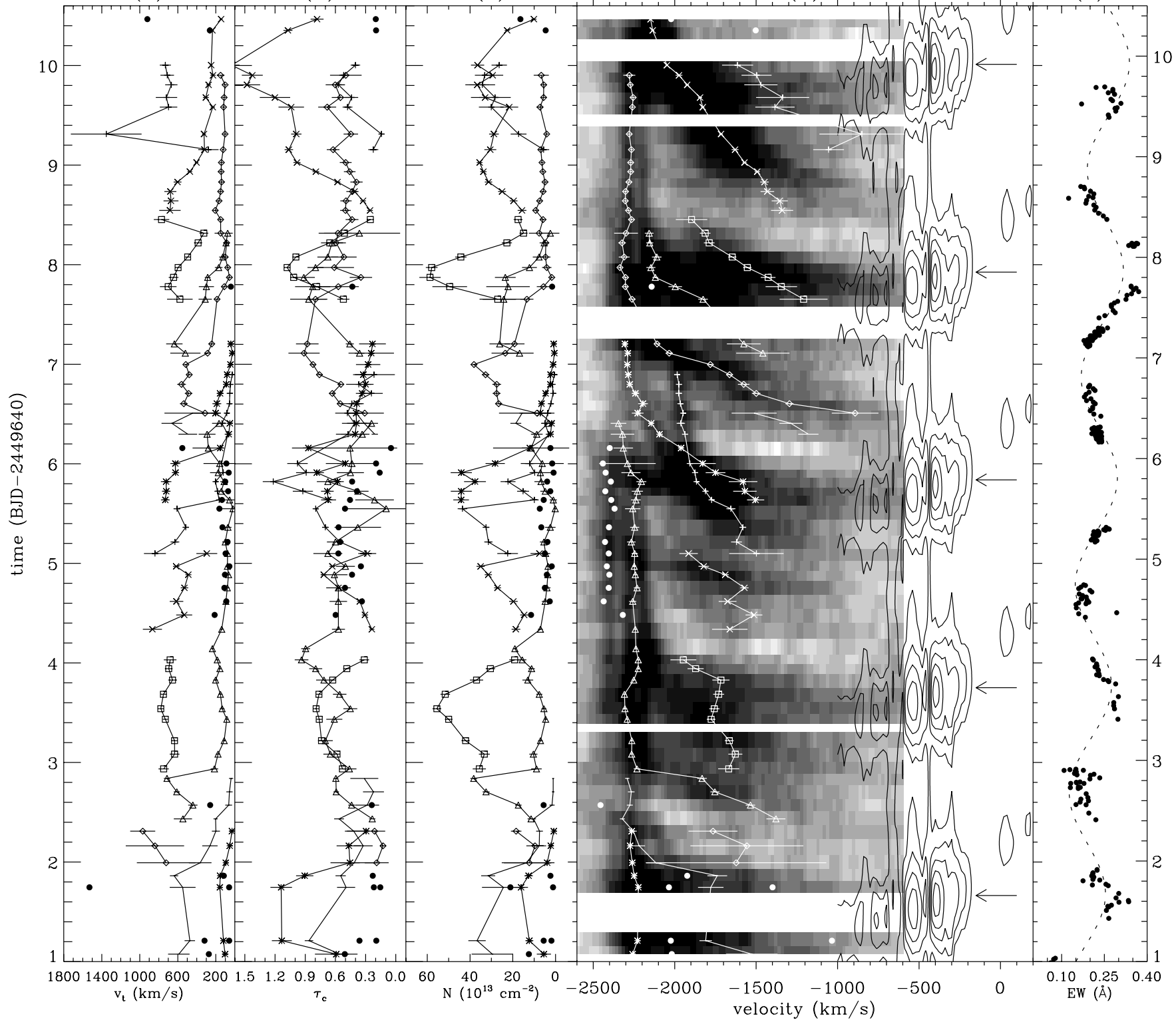

Fig. 9. DAC model fit parameters compared to the variability in N IV and $\mathrm{H} \alpha$. In panels a), b) and $\mathbf{c}$ ) the fit parameters $v_{\mathrm{t}}$ and $\tau_{\mathrm{c}}$ and the column density $N$ are shown, respectively. In panel $\mathbf{d}$ ) the measured central velocity of the DAC is overplotted on the dynamic spectrum of Si IV. The contour plot in the same panel shows the absorption features in N IV (same inverse discrete Fourier transform (IDFT) as in Fig. 16). The $E W$ of $\mathrm{H} \alpha$ between $[-500,-200] \mathrm{km} \mathrm{s}^{-1}$ is shown in panel e). The arrows in panel d) indicate the times of maximum $E W$ in $\mathrm{H} \alpha$, which follow from the sine+trend fit in panel e). The grey-scale conversion is the same as in Fig. 4. The overall hollow curvature in panel $\mathbf{d}$ ) that becomes apparent when one tries to connect the N IV absorption events with the Si IV DACs, is called phase bowing (see Sect. 6)

in de Jong et al. (1999), whereas the error calculation is based on Schwarzenberg-Czerny (1996).

The resulting values of the period $P=1 / f_{0}$ and the corresponding $1-\sigma$ errors $\epsilon_{\mathrm{P}}=\epsilon_{\mathrm{f}} / f^{2}$ are given in Table 5 . Except for the He I line and the edges of the SiIV, NV and $\mathrm{CIV}$, all periods are the same within a $2-\sigma$ confidence limit. The weighted average period of the five similar lines is $P_{1}=2.087(5) \mathrm{d}$.

Some other periods are also present. The Si IV analysis shows a peak at $P_{2}=1.021(3) \mathrm{d}$ and NIV has within the errors a similar period. In addition, $P_{1}$ and $P_{2}$ are not present in the $E W$ of $\mathrm{H} \alpha$ and He I between -200 and $200 \mathrm{~km} \mathrm{~s}^{-1}$, and also not in the edges of the UV lines. From the Fourier analysis follows a period of 6.4 days in the edge variability (see Table 5). This period probably reflects the gradual change in the edge, since the total length of the campaign was only 10 days.

Sinusoids with the $2.09 \mathrm{~d}$ period were fitted through the $E W s$ of $\mathrm{H} \alpha \mathrm{NIV}, \mathrm{Si} I V, \mathrm{CIV}$ and Nv. The times of the maxima are given in Table 5 . The $E W$ maxima of $\mathrm{H} \alpha$ lag $0.26(5) \mathrm{d}$ behind the maxima of Si IV, but coincide with the $E W$ maxima of $\mathrm{C}$ IV and $\mathrm{N} v$. We will later 


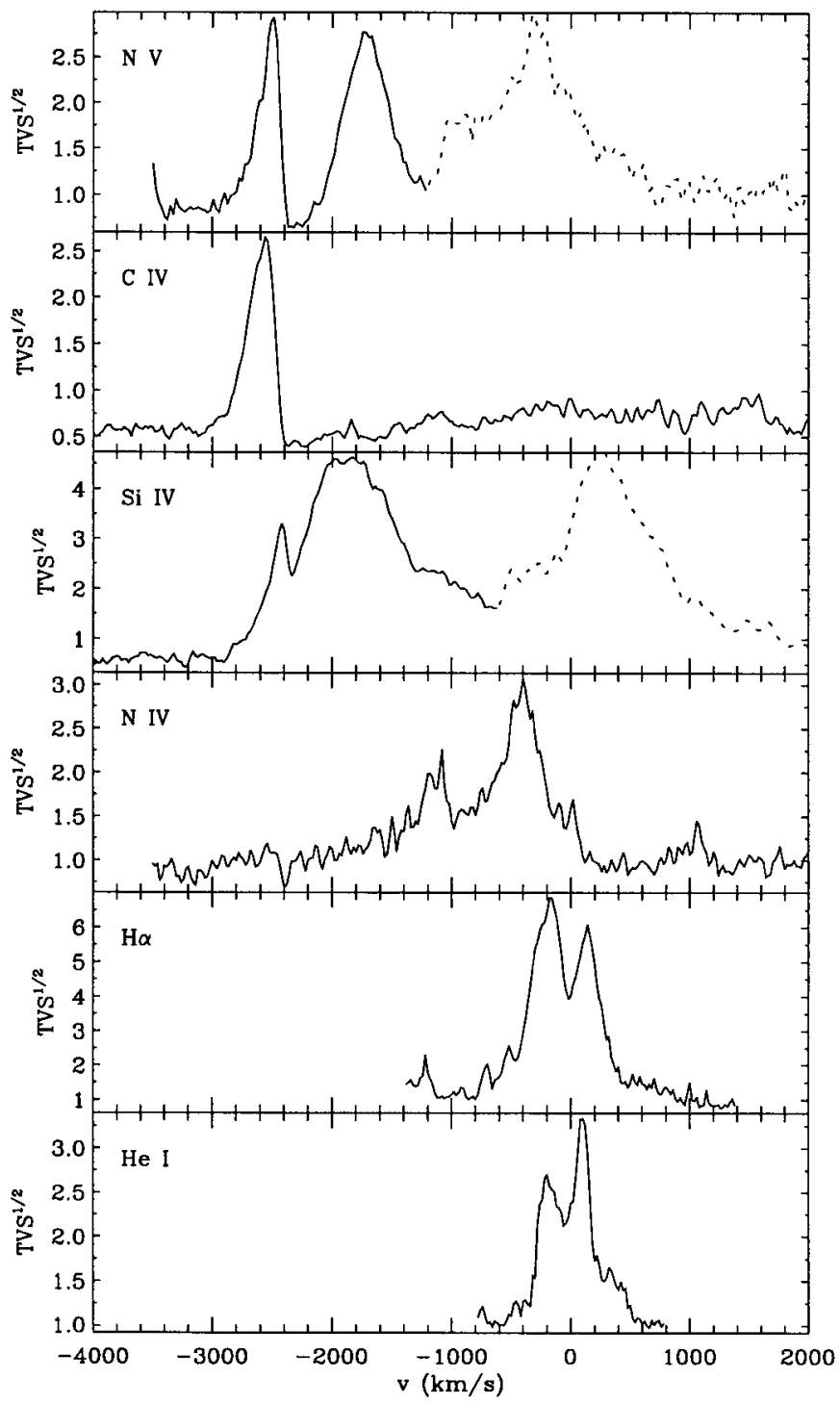

Fig. 10. Temporal variance spectra $(T V S)$ for 4 UV lines (top) and 2 optical lines (bottom). Moving from bottom to top we see that the peak variability moves progressively towards more negative velocities. The Si IV and N v lines also show variability due to the red component of the doublet

discuss these important phase differences in view of the CIR model.

\subsubsection{Periods in line profiles}

In addition to the period analysis of the integrated line profiles, we carried out such an analysis with the CLEAN method for all the studied lines in each velocity bin. The resulting power is plotted as a function of velocity and frequency in the contour plots in Figs. 12 and 13. These figures show that the $2.09 \mathrm{~d}$ period is present in all $\mathrm{UV}$ lines and that the distribution of the power as a function of velocity is similar to the signal distribution in the TVS (see Fig. 10). The periods and the corresponding uncertainties were calculated with the same method as in Sect. 5.5.1. Data points with a power less than $10 \%$ of the maximum power and/or with deviations too large

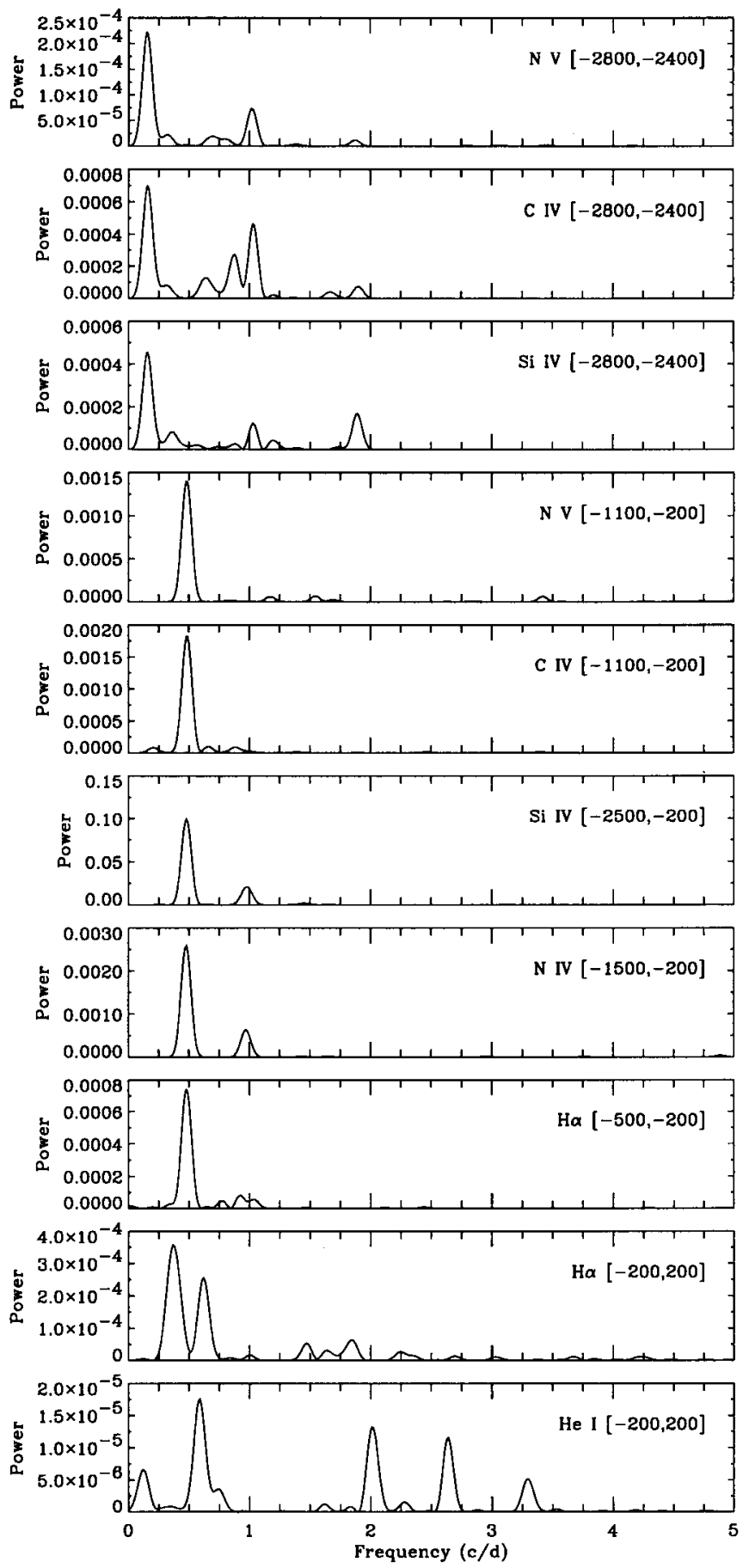

Fig. 11. Search for periodic variability in the $E W$ measurements (see Fig. 8). Shown are the power diagrams produced by the CLEAN analysis. The $2.09 \mathrm{~d}$ period is present in all lines, except for HeI and the edges of the UV resonance lines

compared to their errors were discarded (Chauvenet criterium, see de Jong et al. 1999). The remaining data points are plotted in Fig. 14. The resulting weighted average frequencies are given in the second part of Table 5 . The best determined period is the $2.086(2) \mathrm{d}$ period in the Si IV line, which we adopt as the wind period. All other periods in the $E W$ and lpv's agree with this value within $1 \sigma$ 
Table 5. The main periods found in the periodograms. The columns at the left contain the results of a period analysis based on $E W$ measurements over the intervals indicated. Column 5 gives the epochs when the sinusoid has a maximum. The columns at the right give the results based on a separate period analysis in each velocity bin in the listed interval. The last column gives the weighted average of the calculated periods over the interval. The underlined value is our adopted best-determined period

\begin{tabular}{lllll|llll}
\hline & \multicolumn{3}{c}{ Period analysis of $E W$ measurements } & \multicolumn{3}{c}{ Average of individual velocity bins } \\
Line & Interval & $f$ & $P$ & $t_{\max }$ & Interval & $N_{\text {data }}$ & $f(\mathrm{c} / \mathrm{d})$ & $(\mathrm{d})$ \\
\hline H $\alpha$ & {$[-500,-200]$} & $0.480(8)$ & $2.08(3)$ & $5.84(5)$ & {$[-440,-200]$} & 13 & $0.4829(9)$ & $2.071(4)$ \\
He I & {$[-200,200]$} & $0.59(2)$ & $1.70(6)$ & & & & \\
N IV & {$[-1500,-200]$} & $0.477(7)$ & $2.10(3)$ & $5.62(4)$ & {$[-1220,340]$} & 60 & $0.480(2)$ & $2.083(9)$ \\
& & $0.97(1)$ & $1.03(2)$ & & {$[-1180,-100]$} & 43 & $0.984(5)$ & $1.016(5)$ \\
Si IV & {$[-2500,-200]$} & $0.479(1)$ & $2.087(5)$ & $5.58(1)$ & {$[-2420,780]$} & 143 & $0.4793(4)$ & $2.086(2)$ \\
& & $0.979(3)$ & $1.021(3)$ & & {$[-2240,1240]$} & 152 & $0.958(1)$ & $1.044(2)$ \\
C IV & {$[-1100,-200]$} & $0.485(8)$ & $2.06(4)$ & $5.87(3)$ & {$[-1120,-420]$} & 12 & $0.479(2)$ & $2.086(8)$ \\
N v & {$[-1100,-200]$} & $0.48(1)$ & $2.07(4)$ & $5.86(2)$ & {$[-2200,368]$} & 96 & $0.477(3)$ & $2.09(1)$ \\
Si IV (edge) & {$[-2800,-2400]$} & $0.16(1):$ & $6.4(5):$ & & {$[-2500,-1400]$} & 18 & $0.136(7):$ & $7.3(4):$ \\
C IV (edge) & {$[-2800,-2400]$} & $0.160(9):$ & $6.3(4):$ & & {$[-2580,-2460]$} & 7 & $0.165(7):$ & $6.1(3):$ \\
N v (edge) & {$[-2800,-2400]$} & $0.15(2):$ & $6.5(7):$ & & {$[-2584,-2416]$} & 8 & $0.152(3):$ & $6.6(1):$ \\
\hline
\end{tabular}

(except for the lpv period in $\mathrm{H} \alpha$ ). The difference with the weighted average of the $E W$ period is less than $1 \sigma$.

As can be seen in Fig. 14, the period varies a little as a function of velocity, in particular in Si IV and N IV, where a similar trend can be observed, but in both cases the uncertainties are too large to make it significant. We note in this respect that Fullerton et al. (1997) found a similar trend in the star HD 64760, but could not make it significant either.

\subsection{Comparison of cyclical behaviour in different lines}

In order to search for a connection between the variations in the UV and optical spectra we compared the phases of the periodic modulations. The phase of the signal can be extracted from the CLEANed Discrete Fourier Transform (CDFT) using the following representation:

$F(t)=\Delta f \sum_{i=1}^{N} \sqrt{2 P_{i}} \cos \left(2 \pi\left[f_{i}(t-\bar{t})+\phi_{i}\right]\right)$.

Here $F(t)$ is the flux as a function of time $t, f_{i}$ is the frequency, $\Delta f$ is the frequency interval $f_{i+1}-f_{i}, P_{i}$ is the power, $\phi_{i}$ is the phase of component $i$ and $\bar{t}$ is the average time of the sample. Hereafter we consider only the phase of the component which has the maximum power (at the periods given in Table 5). By convention $\phi$ is defined between 0 and 1 , with $\phi=0$ at a maximum in $F(t)$. Note that features with a larger phase arrive earlier in time. We derived the phases from a multiple least- $\chi^{2}$ cosine fit in which the errors were computed using a Monte-Carlo approach (see de Jong et al. 1999). The time corresponding to the maximum flux in a component, listed in Table 5, is $t_{\max }=\left(N-\phi^{\prime}\right) / f$, with $N$ an integer value between $-\infty$ and $+\infty$ and $\phi^{\prime}=\phi-f \bar{t}$ (from Eq. (8)), which is needed to correct for the different epochs in the datasets.
The phases as a function of velocity derived from the cosine fits are shown in Fig. 15. In case of $\mathrm{H} \alpha, \mathrm{C}$ IV and $\mathrm{N}$ v only the $2.09 \mathrm{~d}$ period was fitted. The N IV and Si IV also contain a $1.02 \mathrm{~d}$ period which was fitted simultaneously. We consider the phases from the cosine fits more reliable than those derived from the CLEAN algorithm, since in the latter method no noise is taken into account. In case of low $S / N$ the CLEAN phases show more correlation between adjacent bins than the cosine fits, and also more than the errors on the fits allow.

In all UV lines the phase is not constant throughout the spectral line, but peaks at a certain velocity: at about $-1400 \mathrm{~km} \mathrm{~s}^{-1}$ for Si IV and N IV. This so-called phase bowing, which is best seen in the Si IV line, is a characteristic for spiral-shaped structures in the wind (see e.g. Owocki 1995). We will discuss this further in Sect. 6 .

We also computed the difference in phase between Si IV and N IV, NIV and $\mathrm{H} \alpha$, and C IV and Si IV. The average phase difference between $\mathrm{H} \alpha$ and the N IV line in the interval $[-460,-200] \mathrm{km} \mathrm{s}^{-1}$ is only $0.005(32)$ cycles, from which we can safely conclude that absorption events occur simultaneously in these lines. However, the absorption events in N IV lead the events in Si IV by about 0.1 cycle in the region between -500 and $-1000 \mathrm{~km} \mathrm{~s}^{-1}$ (see Fig. 15).

To investigate the origin of these phase lags we reconstructed the dynamic spectra by means of an inverse DFT (IDFT), based on selected frequencies only, which contain the 2.09 and $1.02 \mathrm{~d}$ modulation but no noise frequencies. For Si IV and N IV only the fitted cosines of these two periods were used to reconstruct the dynamic spectra. For $\mathrm{H} \alpha$ the CDFT was inverted using all frequencies from 0.1 to $5 \mathrm{c} / \mathrm{d}$ and above the noise threshold. Certainly above $-200 \mathrm{~km} \mathrm{~s}^{-1}$ this line is too complex to describe with a single frequency.

Figure 16 shows the resulting dynamic spectra. In the figure we overplotted symbols indicating the maximum of 
Si IV

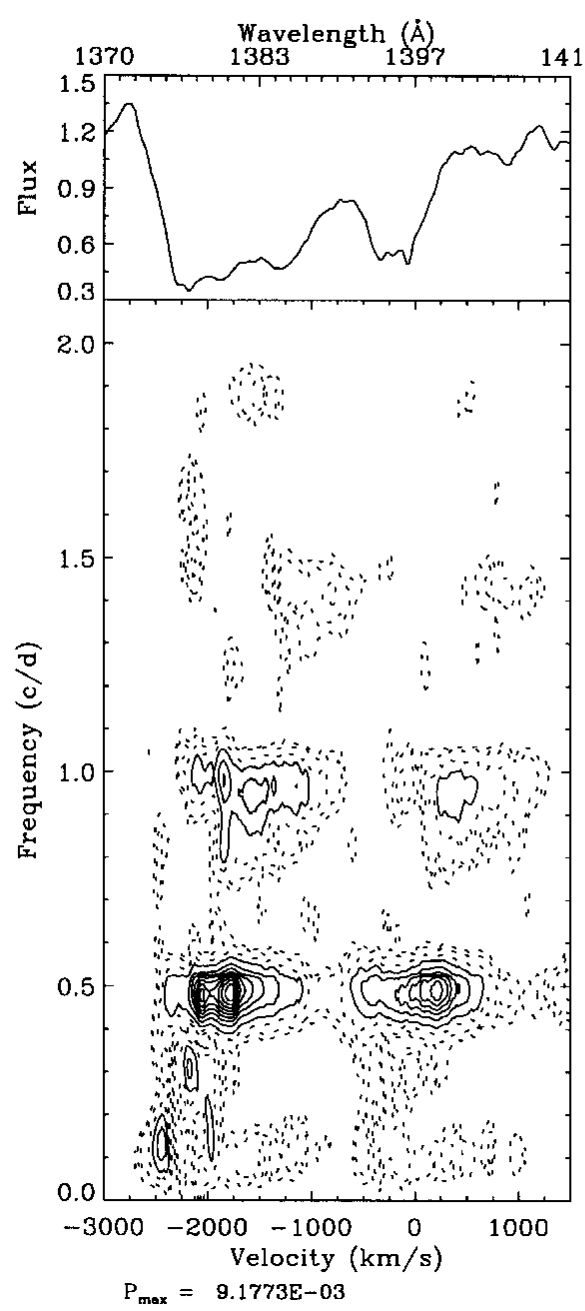

N IV

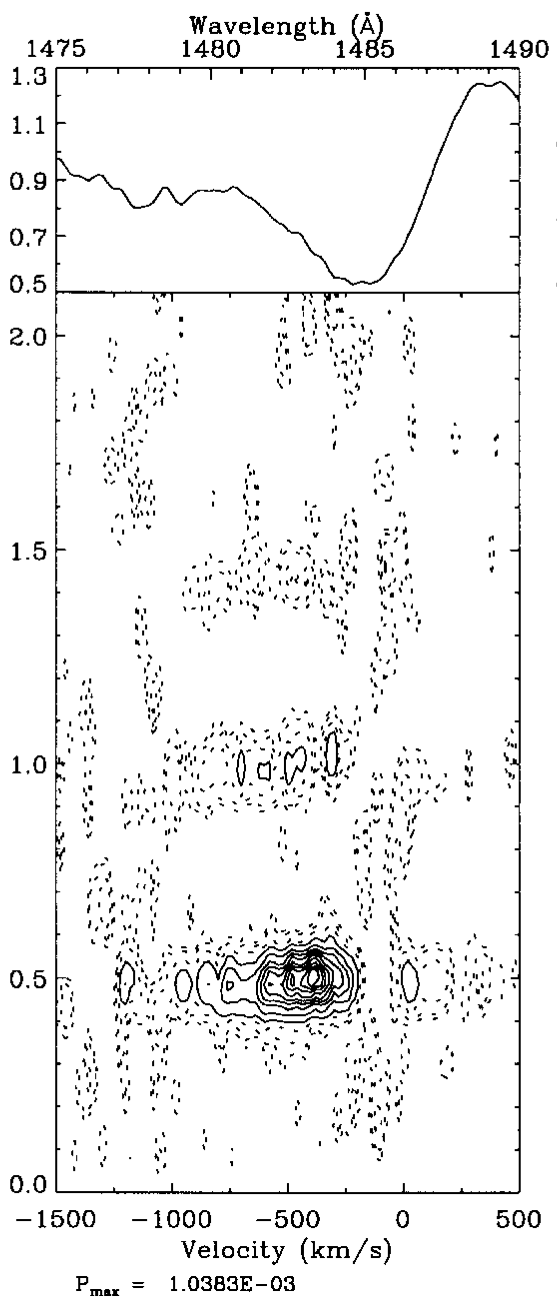

$\mathrm{H} \alpha$

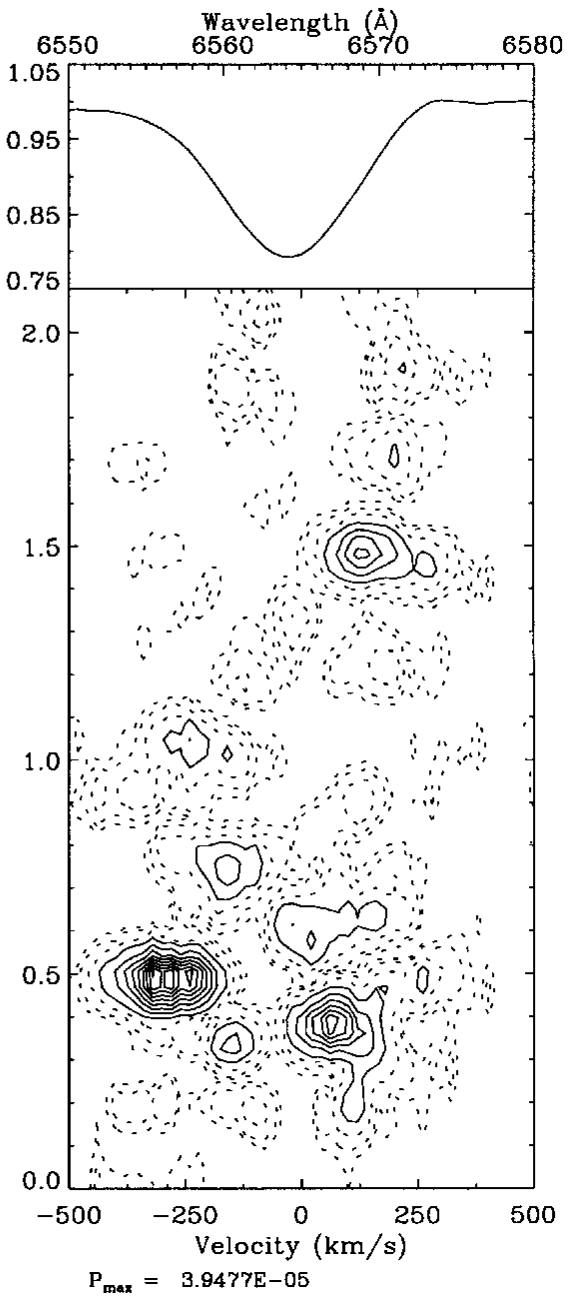

Fig. 12. Power diagrams of Si IV, N IV and H $\alpha$. The upper panels show the normalized average spectrum. The contours indicate the power relative to the highest peak in the diagram, which value is given below each panel. The dashed contours show respectively the $1 \%, 2 \%$ and $5 \%$ levels, while the continuous lines show the levels from $10 \%$ to $100 \%$ (9 levels)

the cosine fits, $t_{\max }$. An interesting phenomenon is visible in this figure. Close inspection of the Si IV panel shows that the DACs consist at least of two components at low velocity, which was already apparent from the DAC fits (see Sect. 5.3 and Fig. 9). We interpret these components as due to separate DACs with different kinematical properties, which merge at higher velocities, yielding a stronger single DAC recurring each $2.09 \mathrm{~d}$. The $1.02 \mathrm{~d}$ period is needed to account for the weak DACs in between. In terms of the CIR model (see below) the different kinematics could correspond to a difference in curvature of the spiral-shaped structures in the wind.

\section{Discussion of the cyclical variability and phase-bowing}

The $2.09 \mathrm{~d}$ period appears in the $\mathrm{H} \alpha, \mathrm{N}$ IV, Si IV, N V and $\mathrm{CIV}$ lines in the velocity range from -200 to $-2420 \mathrm{~km} \mathrm{~s}^{-1}$, which covers the whole wind from the surface of the star up to the regions where the wind has reached its terminal velocity. The relation between the absorption features responsible for this period is especially well shown in Fig. 9 where we superimposed the maximum of the $\mathrm{H} \alpha E W$ between $[-200,-500] \mathrm{km} \mathrm{s}^{-1}$, the N IV absorption features and the DACs of Si IV. All these features closely coincide in time, implying that the prominent DACs in Si IV can be traced down to the surface of the star. Figure 9 also shows that the absorption events are bowshaped. In Si IV the DACs move blue-ward, whereas the absorption components in N IV move red-ward. We have also seen that the phase of the $2.09 \mathrm{~d}$ period is bowed and has a broad peak around $-1400 \mathrm{~km} \mathrm{~s}^{-1}$ (see Kaper et al. 1999a for phase diagrams of $\xi$ Per based on earlier datasets). Thus, according to the Fourier analysis, the Si IV absorption is first detected at about $-1400 \mathrm{~km} \mathrm{~s}^{-1}$ and then splits into a strong blue component (i.e. the accelerating DAC) and a weak red component which moves further to the red. The red component is too faint to be visible in the original spectra, but the effect is clearly seen in the reconstruction shown in Fig. 16. 

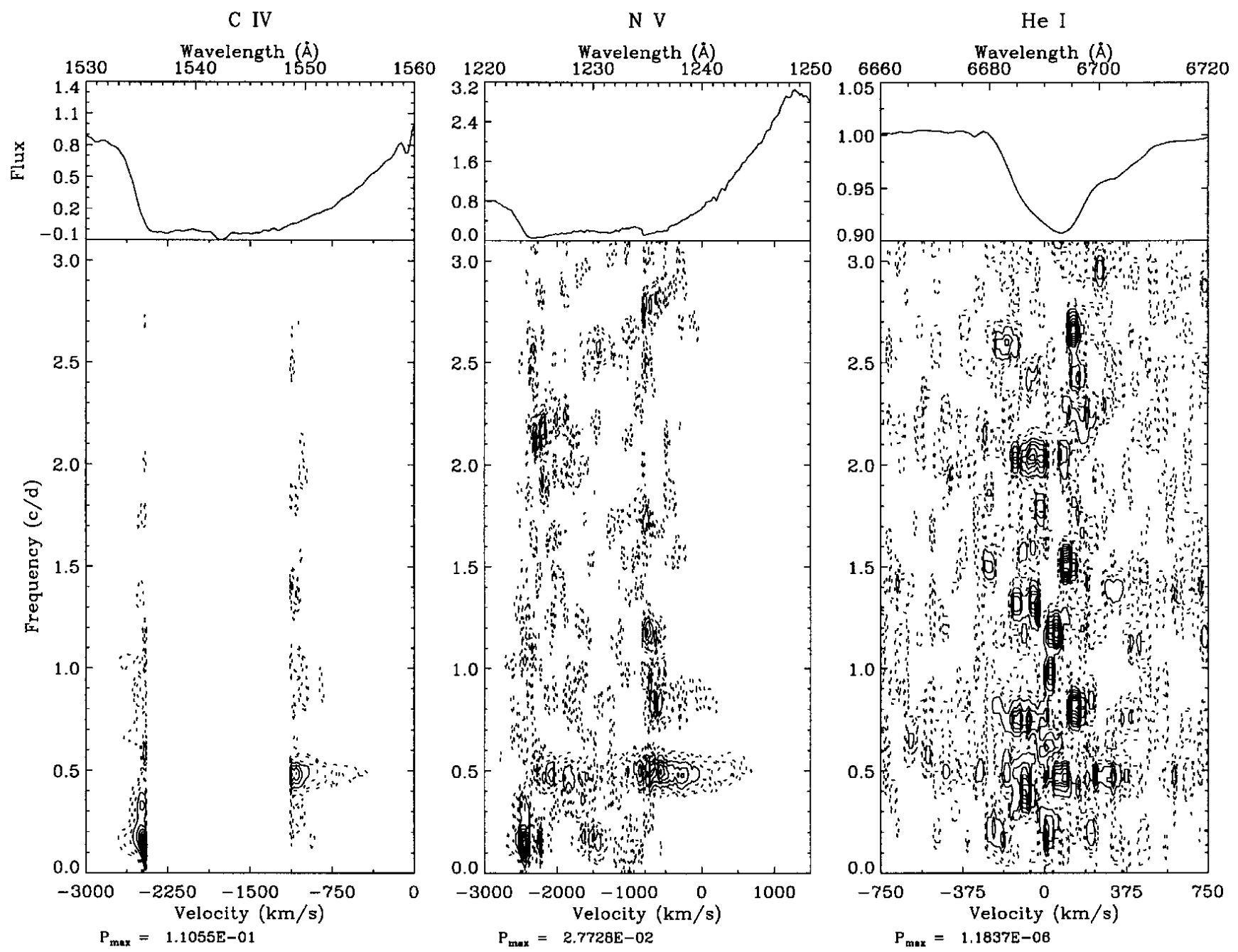

Fig. 13. Powerdiagrams of Nv, CIV and He I. See Fig. 12 for a description

We have to keep in mind that the DAC modeling with Gaussian fits as described in Sect. 5.3 is a phenomenological description which does not specify the actual place and shape of the structures in the wind. For this we have to concentrate on the periodic behavior. One of the most important characteristics is the phase bowing, mentioned above, which can be interpreted as a splitting of the periodic absorption features into two components at different velocities. Phase-bowing is not observed in other $\mathrm{O}$ stars (Kaper et al. 1999a), probably because the DACs are too weak at lower velocities for the $\mathrm{O}$ stars in their sample to be observed with the IUE satellite. Another star which shows very prominent periodic variations is the $\mathrm{B}$ supergiant HD 64760, which was observed during the IUE MEGA campaign (see Massa et al. 1995). In this star the main period is $1.2 \mathrm{~d}$ (Prinja et al. 1995). A second period appears at $2.4 \mathrm{~d}$ and the phases as a function of velocity peak at $-710 \mathrm{~km} \mathrm{~s}^{-1}$ for both periods (Fullerton et al. 1997). They found that a given phase of the modulations occurs progressively later for lines that diagnose higher energy processes, which is in agreement with the phase lag of $0.1 \mathrm{~d}$ which we found between N IV and Si IV (see Sect. 5.6).
Owocki et al. (1995) ascribed this variability to banana shaped modulations in the spectrum rather than to migrating DACs. The analysis of the phase behavior in $\xi$ Per points to a similar view. The IDFT of the main periods of $1.02 \mathrm{~d}$ and $2.09 \mathrm{~d}$ (Fig. 16) shows that the phase of the $2.09 \mathrm{~d}$ period clearly follows the bright banana shaped structures in between the DACs. The $1.02 \mathrm{~d}$ period seems to account mainly for the weaker precursors of the strong DACs (modeled in Fig. 9). We may not be able to see this modulation over the full range of velocity, because of the blending at lower velocities with the red component of the doublet. HD 64760 has a lower $v_{\infty}\left(1500 \mathrm{~km} \mathrm{~s}^{-1}\right)$ and therefore less blending in the Si IV line as compared to $\xi$ Per. Taking all the similarities together there is sufficient ground to conclude that the modulations in HD 64760 and the DACs in $\xi$ Per are similar phenomena. Finally, we note that there were also DAC events observed in HD 64760 with a long period $(9.8 \mathrm{~d})$ with the classical pattern of acceleration (Fullerton et al. 1997). There seems to be no analogy to these events in $\xi$ Per, in which the only indication of a timescale longer than our observation run is found in the edge variability of the UV lines. 

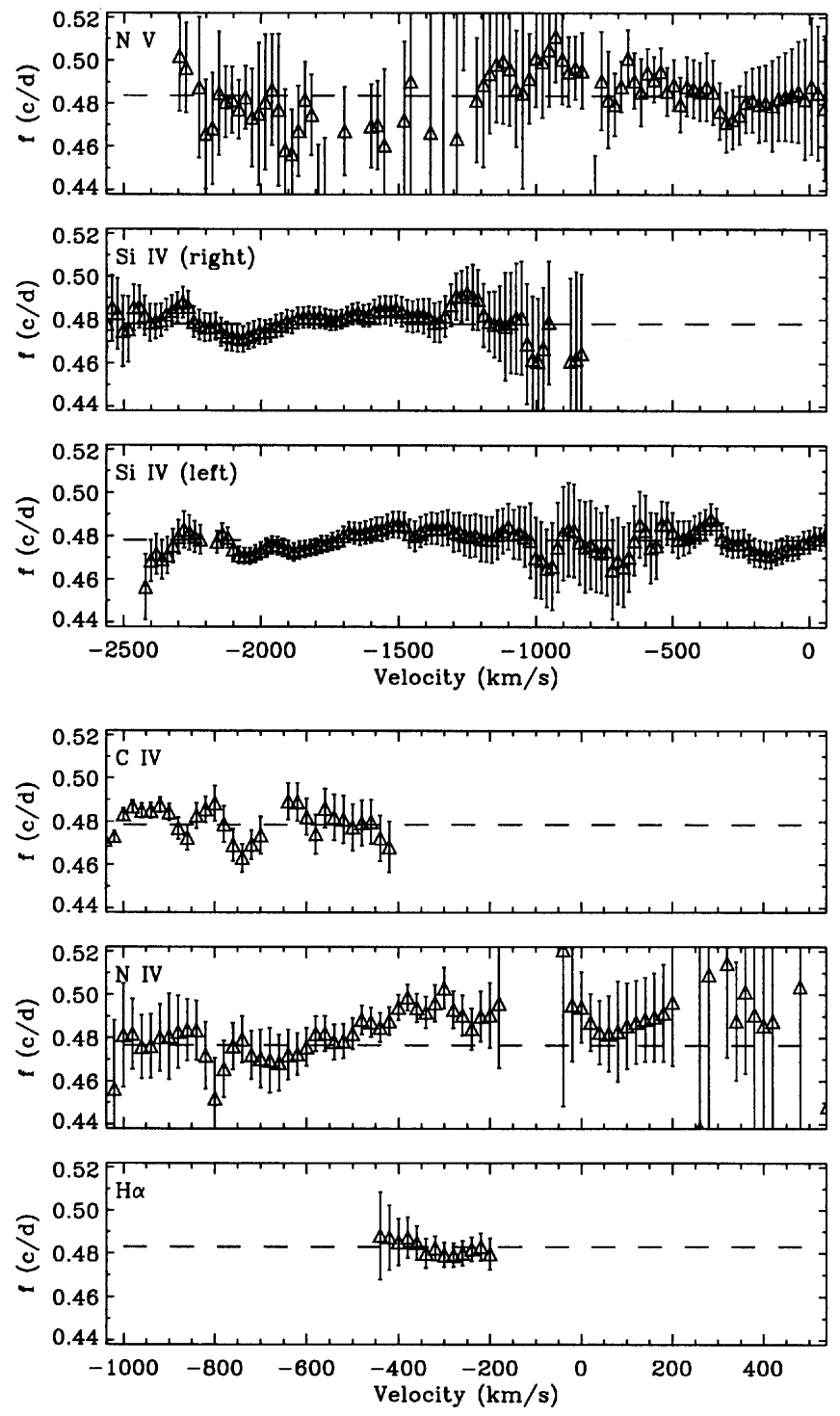

Fig. 14. Measured frequency per wavelength bin obtained in the Fourier spectra by means of Gaussian fits centered at $2.09 \mathrm{~d}$. The dashed lines show the average of all points. Only the fits with a power of at least $5 \%$ of the maximum power are shown

\subsection{Long-term behavior}

Considering the unknown origin of the variability, it is important to investigate the long-term behavior of DACs in $\xi$ Per. The DAC modeling described in Sect. 5.3 shows only the blue-ward moving components and gives results similar to the UV observations from 1987, 1988 and 1991 (Kaper et al. 1999a). These observations also show that strong DACs appear about every 2 days with a weak DAC in between. The DAC parameters at these epochs are comparable: $N_{\text {col }}$ varies between 3.6 and $5.510^{14} \mathrm{~cm}^{-2}$, which is about the same in our observations. The $v_{\mathrm{t}}$ and $\tau_{\mathrm{c}}$ values are similar as well. Kaper et al. derived a period of $2.0 \pm 0.2 \mathrm{~d}$ which is in good agreement with our observations. From this we conclude that irrespective of the physical cause of the DAC events, it must be stable for

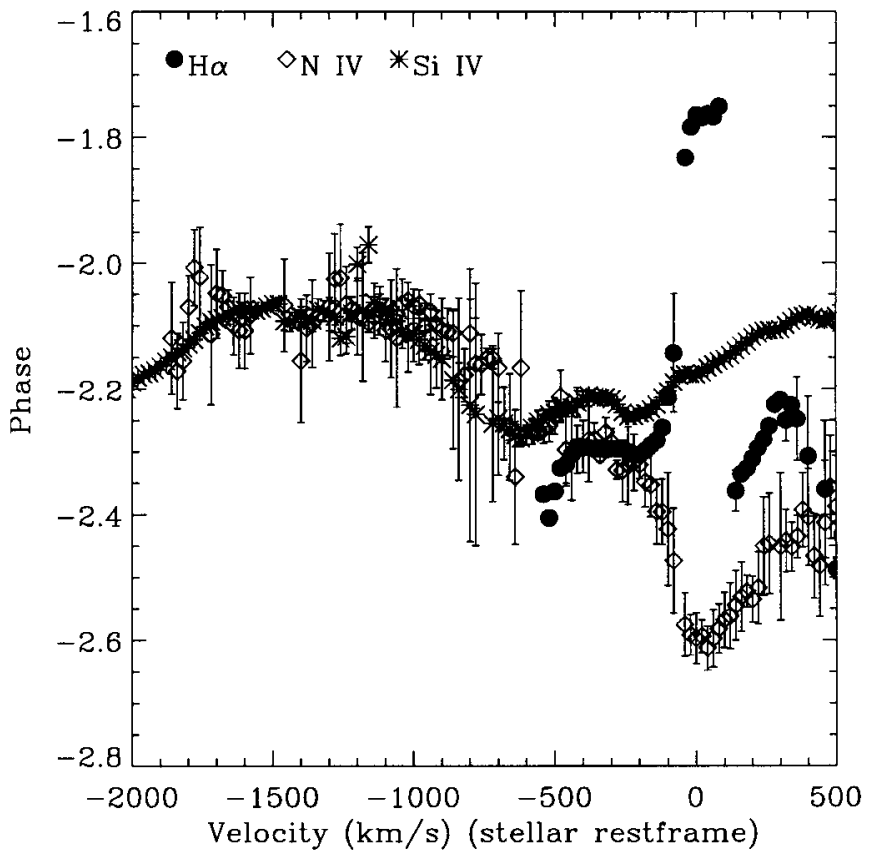

Fig. 15. Phases for the $\mathrm{H} \alpha, \mathrm{N}$ IV and Si IV lines as defined in Eq. (8) and corrected for the different epochs (see Sect. 5.6). In the interval $[-460,-200] \mathrm{km} \mathrm{s}^{-1}$ the average phase difference between $\mathrm{H} \alpha$ and $\mathrm{N}$ IV is only $0.005(32)$. Note the phase bowing in the Si IV lines

at least 7 years. The detailed differences between different years are not larger than the differences between successive DAC events.

\subsection{Modeling}

Currently, the cyclical wind variations which we described above are best explained by the CIR model in which the emergent wind flow is perturbed by some structure at the surface of the star. Such a structure causes a local change in the radial flow properties. Due to the stellar rotation such a stream is curved. Further-out in the wind, slow and fast moving streams collide and form a shock-front that corotates with the star. This model was first proposed by Mullan (1984) in analogy with the CIRs in the solar wind. Owocki et al. (1995) showed that spiral shaped corotating structures give a good explanation for the observed bow-shaped isoflux contours in HD 64760. They used a kinematic model in which spiral "streak" lines are rotated through the line of sight. They showed that the part from the spiral at intermediate velocities enters the absorption column first, followed simultaneously by the low- and high-velocity parts. The low-velocity part ("head") causes the red-ward moving absorption while the high-velocity part ("tail") causes the blue-ward moving absorption simultaneously. The CIR model was worked out in detail and simulated with hydrodynamical computations by Cranmer \& Owocki (1996). Fullerton et al. (1997) made another kinematic approach for HD 64760, which agreed even better with the hydrodynamical model. In order to 
better understand the variability in $\mathrm{H} \alpha$ we made a semiempirical model in which all kinds of combinations of corotating structures can be included. A detailed description of this model is given in De Jong et al. (2001b). We use this model here to simulate the DAC behavior in the UV resonance lines of $\xi$ Per.

Before we can apply our model, we must know the rotation period of $\xi$ Per. It seems most obvious to take for this the most prominent period of $2.09 \mathrm{~d}$. However, it turns out that successive DACs are not identical over this time interval. Especially the alternating column density (see Sect. 5.3 and Fig. 9, panel (c)) is a strong indication that the actual rotation period is twice as long, namely 4.18 d. De Jong et al. (1999) show that this is in agreement with the stellar parameters derived by Puls et al. (1996). With $v_{\text {rot }} \geq 204 \mathrm{~km} \mathrm{~s}^{-1}$ (=v sin $i$, Penny 1996) the radius must be at least $17 R_{\odot}$ and only their model with $M=60 M_{\odot}$ and $R=25 R_{\odot}$ complies with this. Leitherer (1988) derives a smaller radius of $12 R_{\odot}$, which we cannot exclude at this stage. If we adopt $P_{\text {rot }}=4.18 \mathrm{~d}$, this means that the corotating structure of $\xi$ Per must contain at least 2 strong CIRs and 2 weaker ones in order to explain the strong and weak DACs observed within one cycle. We have computed such a model from which the structure is shown in Fig. 17 and the dynamic spectrum in Fig. 18. This dynamic spectrum resembles the observations quite well. The DACs start as weak and broad components, accelerate and become deeper and sharper. The banana-shaped isoflux contours are also present. However, our model does not include any perturbations to the velocity field. Cranmer \& Owocki (1996) found that the largest absorption takes place where the gradient in the velocity is lowest in a so-called "kink" in front of the CIR. This would mean that the maximum absorption from their model will occur earlier in time compared to our model.

\section{Discussion}

\subsection{The CIR model}

Nearly all variations observed in the wind down to the surface of the star can be explained by the presence of CIRs. However, the physical nature of the surface structure causing the CIRs is by no means clear. In this observing run we have only the $\mathrm{H} \alpha$ and $\mathrm{He}$ I $\lambda 6678$ lines that provide information on what happens near the stellar surface. The variations in these line profiles cannot be fit with a simple CIR model. The $2.09 \mathrm{~d}$ period in $\mathrm{H} \alpha$ is associated with the absorption features only between -440 and $-200 \mathrm{~km} \mathrm{~s}^{-1}$, i.e. in the wind. The periodogram of this line reveals no obvious periodic variations at higher velocities, but contains a collection of a number of weak maxima, which cannot easily be linked to regular variability (Fig. 12). The same can be said of the He I line (Fig. 13).

The $\mathrm{H} \alpha$ line also behaves quite differently compared to other years. During the MUSICOS campaign in November 1996 (Henrichs et al. 1998b) there are no clear absorption events in the blue wing, but only red-ward moving absorption and emission events around the center of the line. Similar events were observed in the He I $\lambda 5876$ line. The period analysis indicates that the longest timescale of variability is comparable to the length of the campaign. The highest peak does not belong to the $2 \mathrm{~d}$ period. In other $\mathrm{H} \alpha$ observations made in October 1991 a period of $2.0(1) \mathrm{d}$ is present between -200 and $+50 \mathrm{~km} \mathrm{~s}^{-1}$ where most of the variations occurred (Kaper et al. 1997). In observations of December 1998 a period of 2.06(4) d appears predominantly in the red wing (de Jong et al. 2001a). The absorption in the blue wing was apparently much stronger in October 1994 than in other years in which $\xi$ Per was observed.

We conclude that the timescale of the non-periodic variability in the optical lines is shorter than what is observed in the UV lines. This is probably because the formation of $\mathrm{H} \alpha$ is much more complicated than of the UV lines. The effects of small changes in the wind structure may have a relatively much larger impact on the $\mathrm{H} \alpha$ lineprofile variations due to projection effects, cancellation of absorption and emission and the relatively small region in which $\mathrm{H} \alpha$ is formed. In $\xi$ Per the $\mathrm{H} \alpha$ line is probably formed only up to $1.4 R_{*}$ (de Jong et al. 2001b). Stochastic variations due to clumping may also play a role. In view of this it is interesting to note that the minimum of the edge $E W$ at $M B J D=8$ coincides well with the maximum $E W$ of $\mathrm{H} \alpha$ in the $[-500,-200] \mathrm{km} \mathrm{s}^{-1}$ interval. In $\xi$ Per this holds only for one event, but we note that in the O6I(n)fp star $\lambda$ Cep a correlation between the He II $\lambda 4686$ (formed close to the star) and C IV edge (far out in the wind) was observed in Oct. 1989 during the whole observation run (Henrichs \& Kaper 1990). According to the CIR model there should be a considerable phase lag between these regions. The correlation may be explained by assuming that the instability can accelerate material close to the star up to velocities which are comparable with the terminal velocity, thereby causing variability in the edge of the UV lines.

\subsection{Non-radial pulsations and surface magnetic fields}

We have investigated the presence of non-radial pulsations (NRP) and/or magnetic fields in $\xi$ Per, which are the two most likely causes for azimuthal asymmetry at the stellar surface.

A NRP with a period of $3.45(2) \mathrm{h}$ and mode $\ell=3$ was detected in a set of high time resolution spectra of the He I $\lambda 4713$ line taken in October 1989 (de Jong et al. 1999). Some of the red-ward moving features in the He I $\lambda 6678$ line of Oct. 1994 and in the He I $\lambda 5875$ line of Nov. 1996 (results of the MUSICOS campaign, de Jong et al. 2001a) are both very likely due to this phenomenon. To illustrate this we plotted in Fig. 19 a short timeseries of these lines and overplot the NRP phase maxima for comparison. The close correspondence means that this NRP mode is stable for at least 7 years. This particular NRP mode alone cannot be the cause of the observed cyclic wind variability, 
Si IV

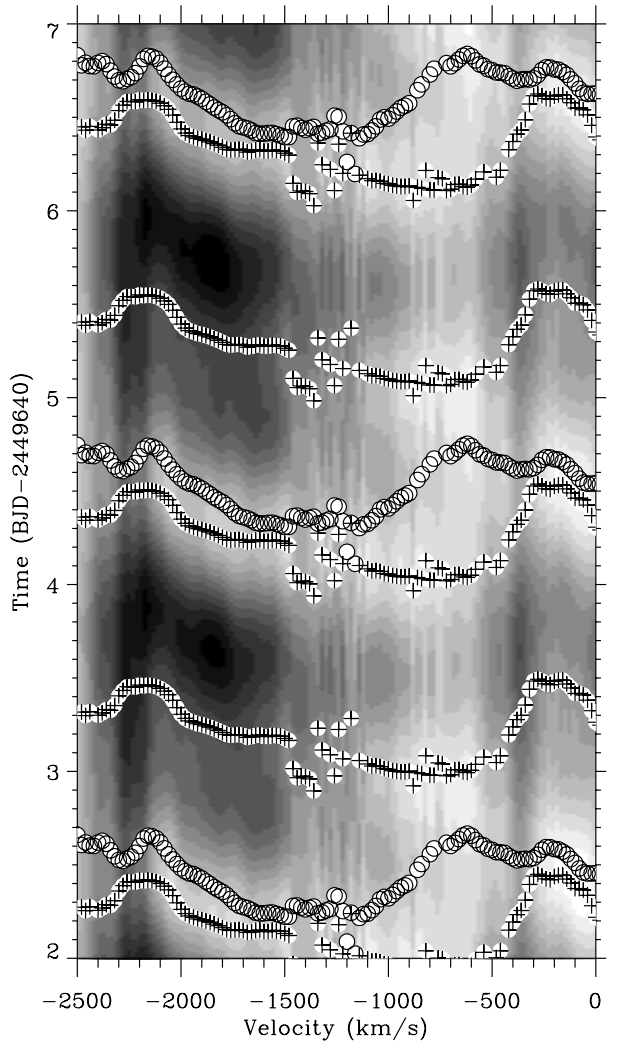

N IV

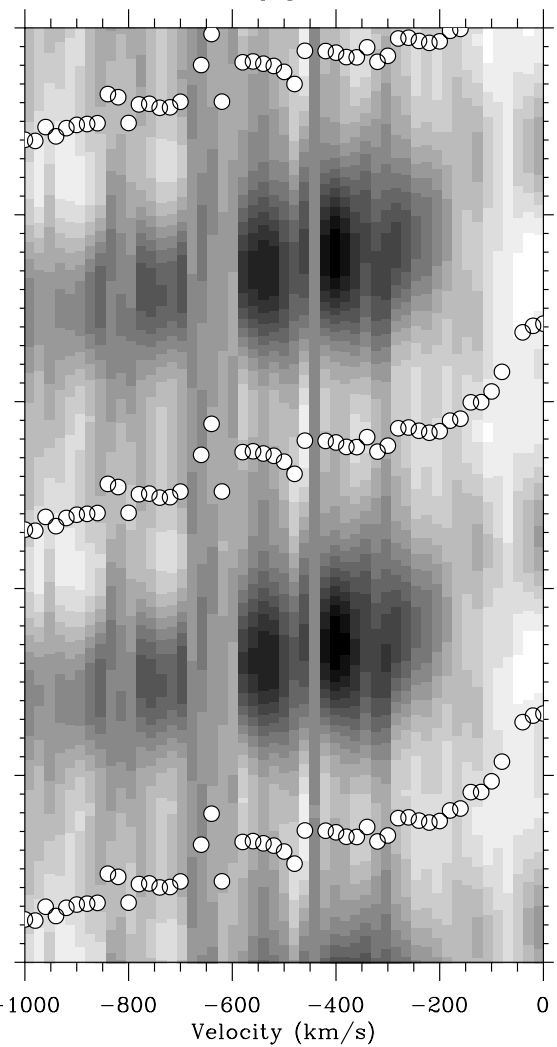

$\mathrm{H} \alpha$

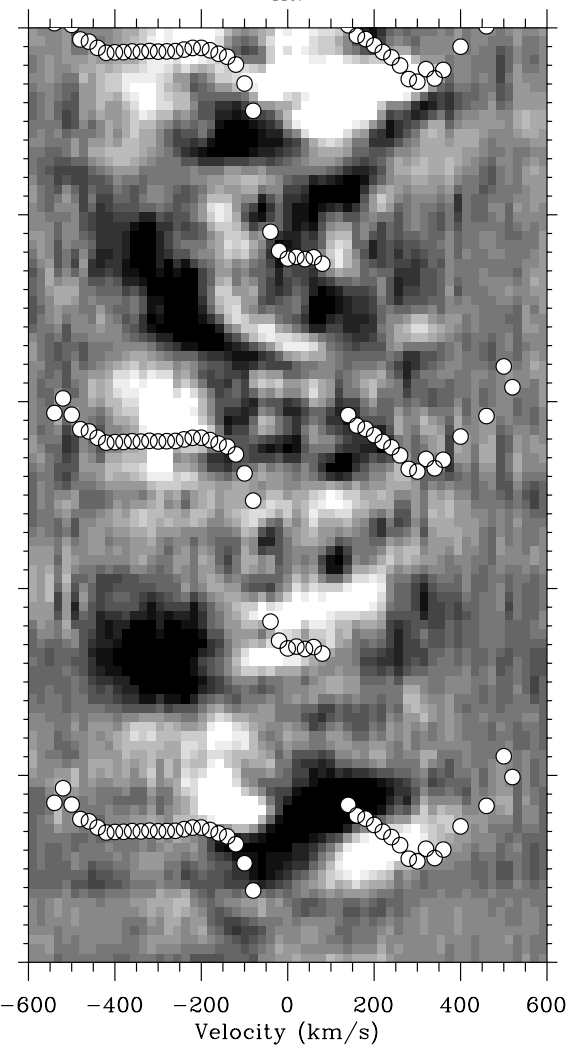

Fig. 16. Inverse discrete Fourier transforms of Si IV, N IV and $\mathrm{H} \alpha$. The overplotted symbols indicate where the maxima of the cosine fits occur. Circles denote the $2.09 \mathrm{~d}$ period, whereas plus signs represent the $1.02 \mathrm{~d}$ period. This figure shows that the phase bowing primarily traces the bright banana shaped features in between the DAC events. The H $\alpha$ line shows much more complex variations which are not traced by the phases

since the pattern speed is too high to be compatible with the wind period of $2.09 \mathrm{~d}$. With $P_{\text {rot }}=4.18 \mathrm{~d}$ the corotating pulsation frequency is $6.2 \mathrm{c} / \mathrm{d}$. If a "bright spot" with a CIR is associated with a certain pulsation phase, we would expect 6.2 CIRs per day crossing the line sight for an equatorial observer. This frequency is too high to be compatible with the wind period of 2.1 days, although we cannot exclude that beating of several (not yet detected) pulsation modes may provide the low-frequency wind modulation. We think that the latter case is unlikely, however, because the main DAC frequency of all investigated $\mathrm{O}$ stars scales with $v \sin i$, whereas the (unknown) beating frequencies will not bear such a relation.

The magnetic field measurements presented here are not conclusive. The upper limit of $70 \mathrm{G}$ in the discaveraged longitudinal component of the field cannot exclude the presence of a field that is strong enough to perturb the flow. If we assume a dipole field which is tilted $90^{\circ}$ with respect to the rotational axis which has an inclination angle of $40^{\circ}$ to the line of sight (de Jong et al. $1999)$ then the polar field strength $\left(B_{\mathrm{p}}\right)$ is about 6 times as large as the measured disk averaged longitudinal component of the field (de Jong et al. 2001a). For a definite detection of a field the strength must be larger than $3 \sigma$, which means that $B_{\mathrm{p}}$ is larger than $1200 \mathrm{G}$. Such a field is more than strong enough to cause the CIRs. Magnetic fields are

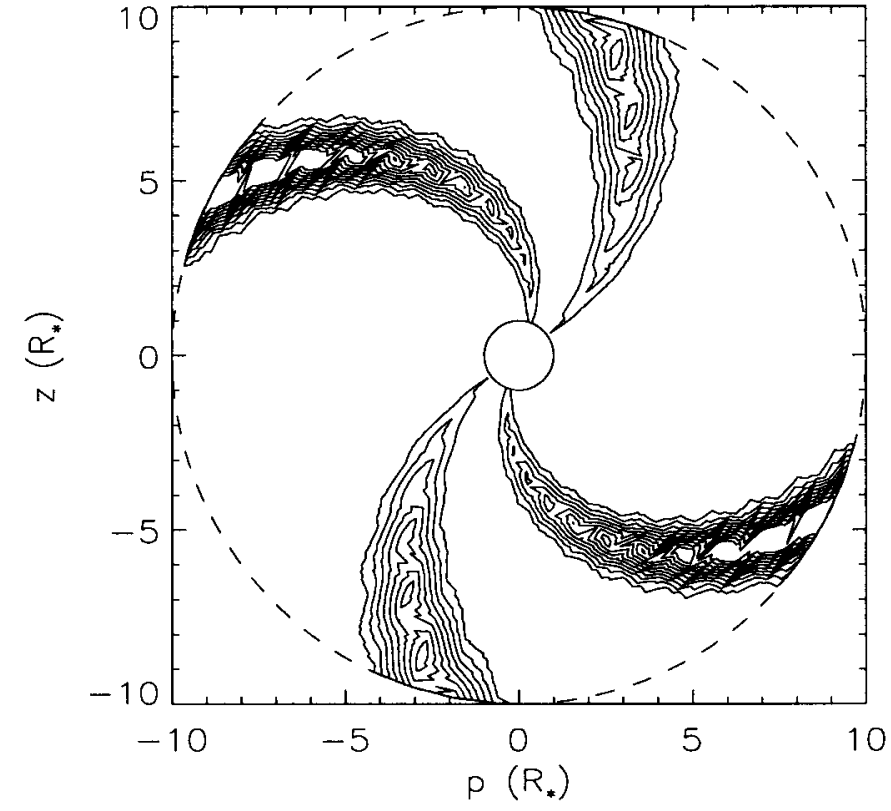

Fig. 17. Corotating structure which was used for synthesizing the dynamic spectrum in Fig. 18

therefore still the most likely candidate for the wind variability, although such fields remain to be detected in $\mathrm{O}$ stars. For other attempts for $\xi$ Per in November 1996 and 


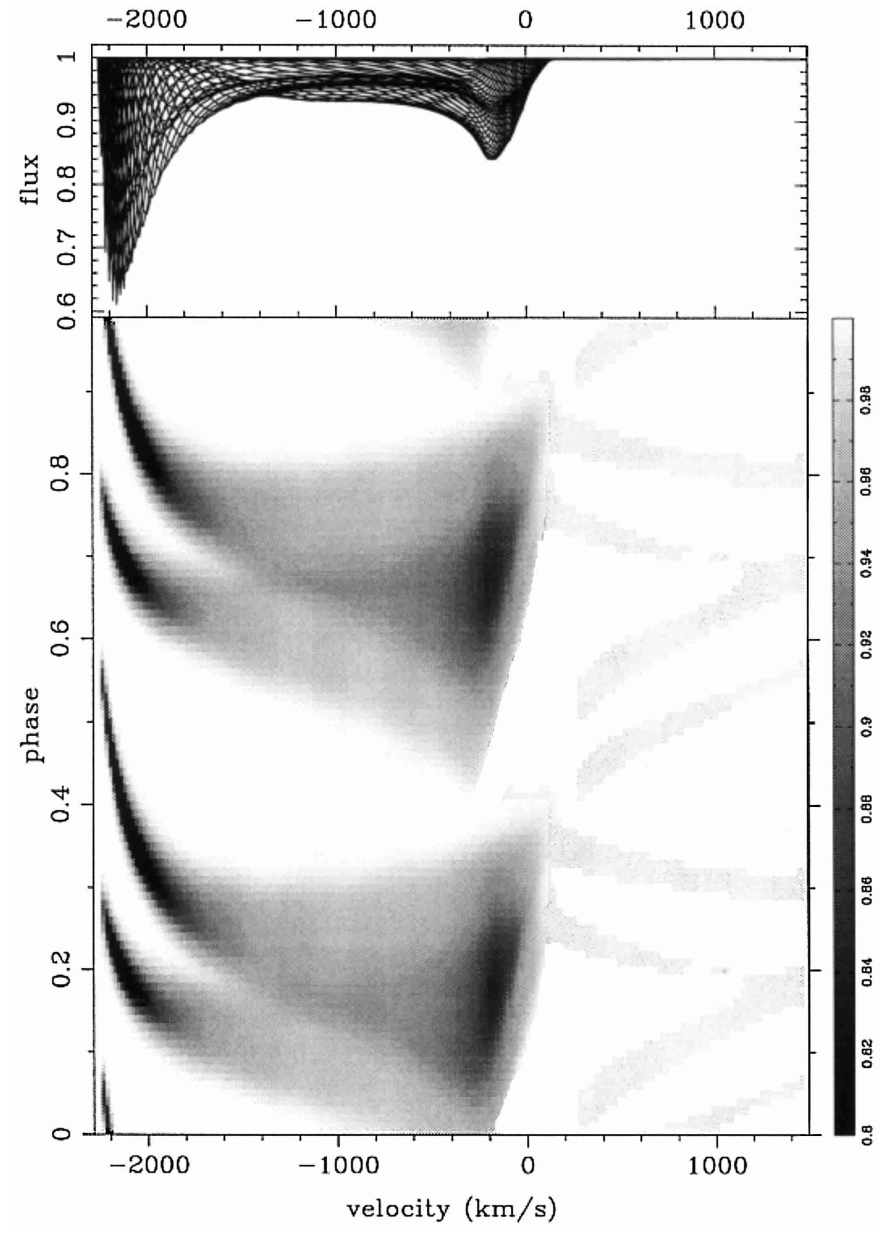

Fig. 18. Generated dynamic spectrum of the simplified CIR model for $\xi$ Per

December 1998 see Henrichs et al. (1998b) and de Jong et al. (2001a). For these attempts we have used the most sensitive instrument available today and obtained only upper limits. More sensitive instrumentation or techniques are apparently needed. We mention that in the B1 IIIe star $\beta$ Cephei (Henrichs et al. 2001) we recently found a sinusoidal varying magnetic field with a semi-amplitude of $90 \mathrm{G}$, which dominates the wind modulation, although differently than in O stars. More research in this direction is clearly needed before the enigma of the cyclical wind variability in early-type stars can be considered to be solved.

\section{Conclusions}

Our main conclusion can be summarized as follows. The entire wind of $\xi$ Per varies with a period of $2.09 \mathrm{~d}$, as shown by the same phase behavior of features formed far out in the wind (DAC's in the UV) to close the stellar surface $(\mathrm{H} \alpha)$, implying that the prominent DACs can be traced down to the surface of the star. The absorption events are bow-shaped (see Figs. 9, 15 and 16). This kind of variability looks similar to the prominent periodic variations in HD 64760, although Owocki et al. (1995) ascribed
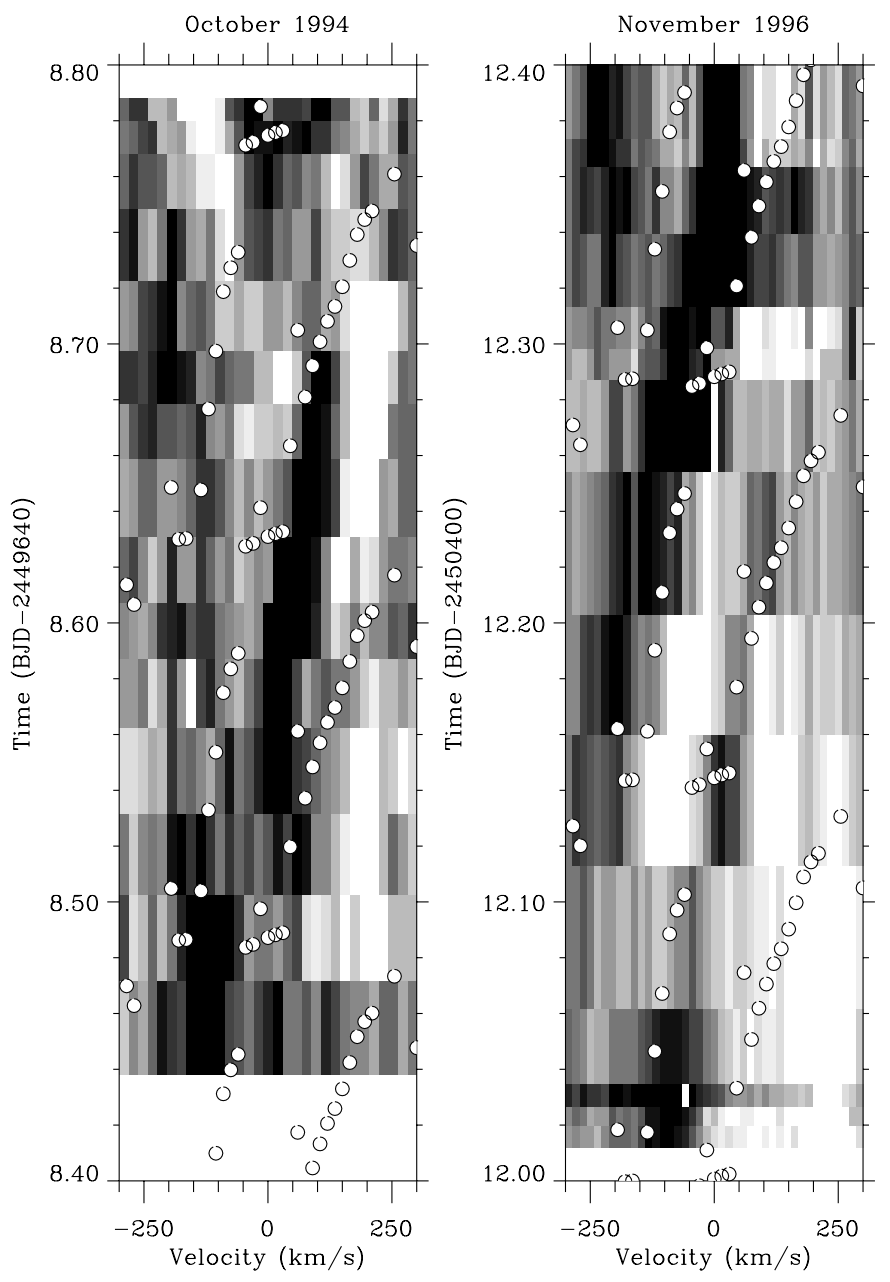

Fig. 19. NRP line-profile variations in the $\xi$ Per observations of Oct. 1994 (this paper) and Nov 1996 (see Henrichs et al. 1998b). The phase of the $3.45 \mathrm{~h} \mathrm{NRP} \mathrm{is} \mathrm{overplotted} \mathrm{with} \mathrm{white}$ circles

this to banana-shaped modulations in flux rather than to migrating DACs. In Sect. 6 a closer examination of the time-variability per velocity bin revealed that also in $\xi$ Per the profiles vary sinusoidally. We conclude that the CIR model (see Sect. 6.2) applies to $\xi$ Per. This is based on the result of Owocki et al. (1995) that spiral shaped corotating structures are a good explanation for these modulations. The asymmetry between the alternating DACs can be explained by assuming that the rotation period could actually be twice the measured period (i.e. $4.18 \mathrm{~d}$ ).

A corotating interaction region needs a seed disturbance at the surface, whichmight be given by NRPs and/or magnetic fields. From a comparison with previous observations we conclude that the CIRs are stable for at least 7 years (see Sect. 6.1). Our magnetic field measurements resulted in null-detections with a 3- $\sigma$ upperlimit of $210 \mathrm{G}$ (Sect. 5.2). The NRP mode with $P=3.45 \mathrm{~h}$ and mode $\ell=3$ reported before by de Jong et al. (1999) seems to be present again. This period is too short to explain the $2.09 \mathrm{~d}$ modulation, although beating with a still undetected mode cannot be excluded. We think, however, that this is unlikely. The ultimate cause of the 
cyclical wind variability in OB stars is clearly not yet known, and especially new surface magnetic field measurements are needed.

Acknowledgements. We thank the support received at the many observatories involved in this large project. We thank K. Annuk, L. Leedjärv and M. Ruusalepp for their help to observe at Tartu Observatory and S. Prins for her help to observe with the JKT. J. D. Landstreet is gratefully acknowledged for his support in our use of the UWO polarimeter at the CFHT. We thank Rens Waters for his helpful comments. JDJ acknowledges support from the Netherlands Foundation for Research in Astronomy (NFRA) with financial aid from the Netherlands Organization for Scientific Research (NWO) under project 781-71-053. LK is supported by a fellowship of the Royal Academy of Sciences in the Netherlands. G. Hill was Visiting Astronomer of the Canada-France-Hawaii Telescope, operated by the National Research Council of Canada, the Centre National de la Recherche Scientifique of France, and the University of Hawaii.

\section{References}

Ballester, P. 1994, A\&A, 286, 1011

Barker, P. K. 1984, AJ, 89, 899

Carbonell, M., Oliver, R., \& Ballester, J. L. 1992, A\&A, 264, 350

Chalabaev, A., \& Maillard, J. P. 1983, A\&A, 127, 279

Cranmer, S. R., \& Owocki, S. P. 1996, ApJ, 462, 469

de Jong, J. A., Henrichs, H. F., Schrijvers, C., et al. 1999, A\&A, 345,172

de Jong, J. A., Henrichs, H. F., Donati, J.-F., et al. 2001a, in preparation

de Jong, J. A., Kaper, L., Brown, J. C., et al. 2001b, in preparation

Fullerton, A. W., Gies, D. R., \& Bolton, C. T. 1996, ApJS, 103,475

Fullerton, A. W., Massa, D. L., Prinja, R. K., et al. 1997, A\&A, 327,699

Giddings, J. 1981, IUE ESA Newsletter, 12, 22

Gordon, K. 1995, Reduce95 Reduction Manual, Ritter Obs., Univ. of Toledo

Hall, J. C., Fulton, E. E., Huenemoerder, D. P., et al. 1994, PASP, 106, 315
Henrichs, H. F., Hammerschlag-Hensberge, G., Howarth, I. D., \& Barr 1983, ApJ, 268, 807

Henrichs, H. F., Kaper, L., \& Zwarthoed, G. A. A. 1988, in A Decade of UV Astronomy with the IUE Satellite (ESA SP-281), vol. 2, 145

Henrichs, H. F., \& Kaper, L. 1990, IUE Astronomy in the era of new space astronomy, ed. E. Rolfe, ESA SP-310, 401

Henrichs, H. F., Kaper, L., \& Nichols, J. S. 1994, A\&A, 285, 565

Henrichs, H. F., de Jong, J. A., Catala, C., et al. 1998b, Proc. Cyclical variability in stellar winds, ESO workshop October 1997, 374

Henrichs, H. F., de Jong, J. A., Donati, J. F., et al. 2001, in preparation

Horne, K. 1986, PASP, 98, 609

Kaper, L., Henrichs, H. F., Nichols, J., et al. 1996, A\&AS, 116, 257

Kaper, L., Henrichs, H. F., Fullerton, A. W., et al. 1997, A\&A, 327,281

Kaper, L., Henrichs, H. F., Nichols, J. S., \& Telting, J. H. 1999a, A\&A, 344, 231

Landstreet, J. D. 1982, ApJ, 258, 639

Leitherer, C. 1988, ApJ, 326, 356

Massa, D., Fullerton, A. W., Nichols, J. S., et al. 1995, ApJ, 452, L53

Mathys, G. 1989, Fund. Cosmic Phys., 13, 143

Mullan, D. J. 1984, ApJ, 283, 303

Mullan, D. J. 1986, A\&A, 165, 157

Owocki, S. P, Cranmer, S. R., \& Fullerton, A. W. 1995, ApJ, 453, L37

Penny, L. R. 1996, ApJ, 463, 737

Press, W. H., Teukolski, S. A., Vetterling, W. T., et al. 1992, Numerical Recipes in C (Cambridge Univ. Press)

Prinja, R. K., Howarth, I. D., \& Henrichs, H. F. 1987, ApJ, 317,389

Prinja, R. K. 1988, MNRAS, 231, 21P

Prinja, R. K., Massa, D., \& Fullerton, A. W. 1995, ApJ, 452, L61

Puls, J., Kudritzki, R.-P., Herrero, A., et al. 1996, A\&A, 305, 171

Roberts, D. H., Lehar, J., \& Dreher, J. W. 1987, AJ, 93, 968

Schwarzenberg-Czerny, A. 1996, ApJ, 460, 107

Telting, J. H., \& Kaper, L. 1994, A\&A, 284, 515

Underhill, A. B., \& Fahey, P. F. 1984, ApJ, 280, 712 